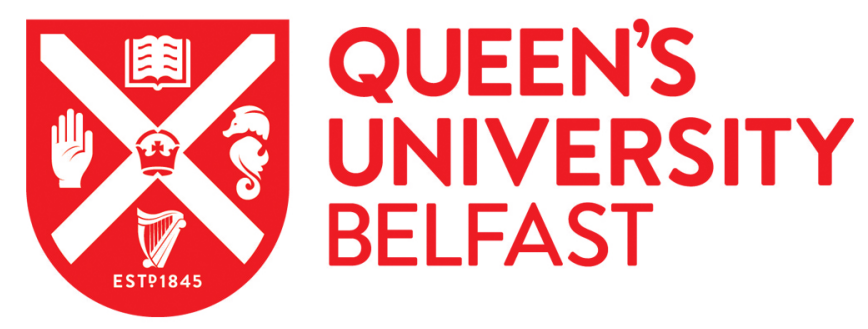

\title{
Absolute magnitudes and slope parameters for 250,000 asteroids observed by Pan-STARRS PS1 - preliminary results
}

Vereš, P., Jedicke, R., Fitzsimmons, A., Denneau, L., Granvik, M., Bolin, B., Chastel, S., Wainscoat, R. J., Burgett, W. S., Chambers, K. C., Flewelling, H., Kaiser, N., Magnier, E. A., Morgan, J. S., Price, P. A., Tonry, J. L., \& Waters, C. (2015). Absolute magnitudes and slope parameters for 250,000 asteroids observed by PanSTARRS PS1 - preliminary results. Icarus, 261, 34-47. https://doi.org/10.1016/j.icarus.2015.08.007

Published in:

Icarus

Document Version:

Peer reviewed version

Queen's University Belfast - Research Portal:

Link to publication record in Queen's University Belfast Research Portal

Publisher rights

(c) 2015, Elsevier. Licensed under the Creative Commons Attribution-NonCommercial-NoDerivatives 4.0 International

$\mathrm{http}: / /$ creativecommons.org/licenses/by-nc-nd/4.0/ which permits distribution and reproduction for non-commercial purposes, provided the author and source are cited.

\section{General rights}

Copyright for the publications made accessible via the Queen's University Belfast Research Portal is retained by the author(s) and / or other copyright owners and it is a condition of accessing these publications that users recognise and abide by the legal requirements associated with these rights.

Take down policy

The Research Portal is Queen's institutional repository that provides access to Queen's research output. Every effort has been made to ensure that content in the Research Portal does not infringe any person's rights, or applicable UK laws. If you discover content in the Research Portal that you believe breaches copyright or violates any law, please contact openaccess@qub.ac.uk. 


\section{Accepted Manuscript}

Absolute magnitudes and slope parameters for 250,000 asteroids observed by

Pan-STARRS PS1- preliminary results

Peter Vereš, Robert Jedicke, Alan Fitzsimmons, Larry Denneau, Mikael

Granvik, Bryce Bolin, Serge Chastel, Richard J. Wainscoat, William S. Burgett,

Kenneth C. Chambers, Heather Flewelling, Nick Kaiser, Eugen A. Magnier, Jeff

S. Morgan, Paul A. Price, John L. Tonry, Christopher Waters

PII:

S0019-1035(15)00351-6

DOI: http://dx.doi.org/10.1016/j.icarus.2015.08.007

Reference:

YICAR 11678

To appear in: $\quad$ Icarus

Received Date: $\quad 2$ June 2015

Please cite this article as: Vereš, P., Jedicke, R., Fitzsimmons, A., Denneau, L., Granvik, M., Bolin, B., Chastel, S., Wainscoat, R.J., Burgett, W.S., Chambers, K.C., Flewelling, H., Kaiser, N., Magnier, E.A., Morgan, J.S., Price, P.A., Tonry, J.L., Waters, C., Absolute magnitudes and slope parameters for 250,000 asteroids observed by PanSTARRS PS1- preliminary results, Icarus (2015), doi: http://dx.doi.org/10.1016/j.icarus.2015.08.007

This is a PDF file of an unedited manuscript that has been accepted for publication. As a service to our customers we are providing this early version of the manuscript. The manuscript will undergo copyediting, typesetting, and review of the resulting proof before it is published in its final form. Please note that during the production process errors may be discovered which could affect the content, and all legal disclaimers that apply to the journal pertain. 


\title{
Absolute magnitudes and slope parameters for 250,000 asteroids observed by Pan-STARRS PS1 - preliminary results.
}

\author{
Peter Vereš ${ }^{1,2}$, Robert Jedicke ${ }^{1}$, Alan Fitzsimmons ${ }^{3}$, Larry Denneau ${ }^{1}$, \\ Mikael Granvik ${ }^{4,5}$, Bryce Bolin ${ }^{1,6}$, Serge Chastel $^{1}$, Richard J. Wainscoat ${ }^{1}$,
}

William S. Burgett ${ }^{7}$, Kenneth C. Chambers ${ }^{1}$, Heather Flewelling ${ }^{1}$, Nick Kaiser ${ }^{1}$,

Eugen A. Magnier ${ }^{1}$, Jeff S. Morgan ${ }^{1}$, Paul A. Price ${ }^{1}$, John L. Tonry ${ }^{1}$, Christopher Waters ${ }^{1}$

veres@fmph.uniba.sk

Received

accepted

\footnotetext{
${ }^{1}$ Institute for Astronomy, University of Hawaii at Manoa, 2680 Woodlawn Drive, Honolulu, HI 96822, USA

${ }^{2}$ Faculty of Mathematics, Physics and Informatics, Comenius University in Bratislava, Mlynská Dolina F1, 84248 Bratislava, Slovakia

${ }^{3}$ Queen's University Belfast, Belfast BT7 1NN, Northern Ireland, UK

${ }^{4}$ Department of Physics, P.O. Box 64, 00014 University of Helsinki, Finland

${ }^{5}$ Finnish Geospatial Research Institute, P.O. Box 15, 02430 Masala, Finland

${ }^{6}$ UNS-CNRS-Observatoire de la Côte d'Azur, BP 4229, 06304 Nice Cedex 4, France

${ }^{7}$ GMTO Corp., 251 S. Lake Ave., Suite 300, Pasadena, CA 91101, USA
} 


\begin{abstract}
We present the results of a Monte Carlo technique to calculate the absolute magnitudes $(H)$ and slope parameters $(G)$ of $\sim 240,000$ asteroids observed by the Pan-STARRS1 telescope during the first 15 months of its 3-year all-sky survey mission. The system's exquisite photometry with photometric errors $\lesssim 0.04 \mathrm{mag}$, and well-defined filter and photometric system, allowed us to derive accurate $H$ and $G$ even with a limited number of observations and restricted range in phase angles. Our Monte Carlo method simulates each asteroid's rotation period, amplitude and color to derive the most-likely $H$ and $G$, but its major advantage is in estimating realistic statistical+systematic uncertainties and errors on each parameter. The method was tested by comparison with the well-established and accurate results for about 500 asteroids provided by Pravec et al. (2012) and then applied to determining $H$ and $G$ for the Pan-STARRS1 asteroids using both the Muinonen et al. (2010) and Bowell et al. (1989) phase functions. Our results confirm the bias in MPC photometry discovered by (Jurić et al. 2002).
\end{abstract}

Subject headings: Solar system, Near-Earth objects, Asteroids, Data Reduction Techniques 


\section{Introduction}

Asteroid diameters are critical to understanding their dynamical and morphological evolution, potential as spacecraft targets, impact threat, and much more, yet most asteroid diameters are uncertain by $>50 \%$ because of the difficulties involved in calculating diameter from apparent brightness. The problem is that an asteroid's apparent brightness is a complicated function of the observing geometry, their irregular shapes, rotation phase, albedo, lack of atmosphere, and their rough, regolith-covered surfaces. Most of these data are unknown for most asteroids. The issue has been further confused because catalogued apparent magnitudes for individual asteroids may have been reported by numerous observers and observatories over many years (even decades) in a variety of photometric systems with varying concern for ensuring accuracy and precision. This work describes our process for calculating asteroid absolute magnitudes (from which diameter is calculated) and their statistical and systematic uncertainties for hundreds of thousands of asteroids using sparse but accurate and precise data from a single observatory, the Pan-STARRS1 facility on Maui, HI, USA. Our technique is suited to estimating absolute magnitudes when the phase curve coverage is even more sparse than those obtained by the Palomar Transient Factory (Law et al. 2009).

An asteroid's absolute magnitude, $H$, is the apparent Johnson $V$ band magnitude, $m$, it would have if observed from the Sun at a distance of 1 au (i.e. observed at zero phase angle and 1 au distance). Accurate measurements of $H$ as a function of time, together with infrared, polarimetric and radiometric observations, can provide crucial information about an asteroid's size and shape, geometric albedo, surface properties and spin characteristics.

In 1985 the International Astronomical Union (IAU) adopted the two-parameter phase function developed by Bowell et al. (1989, hereafter B89), $\Phi_{B}\left(\alpha ; H_{B}, G_{B}\right)$, describing the 
behavior of the apparent magnitude:

$$
m\left(r, \Delta ; H_{B}, G_{B}\right)=5 \log (r \Delta)+\Phi_{B}\left(\alpha ; H_{B}, G_{B}\right)
$$

where $\Delta$ represents the topocentric distance, $r$ the heliocentric distance, and $\alpha(r, \Delta)$ is the phase angle, the angle between the Earth and Sun as observed from the asteroid. We denote absolute magnitude in the B89 system as $H_{B}$ with a corresponding slope parameter, $G_{B}$, that depends in a non-analytical manner on (at least) an asteroid's albedo and spectral type (B89; Lagerkvist and Magnusson 1990). The slope parameter determines how strongly the apparent brightness of an asteroid depends on the phase angle and accounts for the properties of scattered light on the asteroid's surfaces. $G_{B}$ has an average value of $\sim 0.15$ (B89) for the most numerous S and C-class main belt asteroid taxonomies. An accurate determination of both $H_{B}$ and $G_{B}$ requires a wide and dense time coverage of the object's apparent magnitude. Therefore, it is not surprising that only a few tens of slope parameters were measured before the advent of dedicated CCD asteroid surveys.

The B89 phase function was very successful, but observations in the past twenty years have shown it can not reproduce the opposition brightening of E-type asteroids, the linear phase curve of the F-type asteroids, and fails to accurately predict the apparent brightness of asteroids at small phase angles. To address these issues Muinonen et al. (2010, hereafter M10) introduced an alternative phase function, $\phi_{M}$, with two slope parameters, $G_{1}$ and $G_{2}$ that uses cubic splines to more accurately describe the behavior of the apparent magnitude. An alternative M10 formulation with a single slope parameter, $G_{12}$ that is denoted in our work as $G_{M}$, can be used when the data are not sufficient to derive the values of the two-parameter formulation i.e. $m=5 \log (r \Delta)+\Phi_{M}\left(\alpha ; H_{M}, G_{M}\right)$. Their phase function was constructed such that $H_{M} \sim H_{B}$ and the average asteroid would have a slope parameter of $G_{M} \sim 0.5$. This form of the phase function can provide better apparent magnitude predictions but derivation of $H_{M}$ and $G_{M}$ still requires extensive light curve coverage and 
well-calibrated observational data (Oszkiewicz et al. 2012). The IAU adopted the M10 $(H, G 1, G 2)$ system as the new photometric system for asteroids in 2012.

In the remainder of this work we use $H$ and $G$ to represent 'generic' absolute magnitudes and slope parameters respectively, and use the subscripts $B$ and $M$ on each parameter when referring to the values calculated using the B89 and M10 phase functions respectively. We implemented both functions to facilitate comparison with 1) past work that used the B89 parameterization and 2) future work that will use the now-standard M10 implementation. When we use $G_{M}$ we specifically mean the M10 $G_{12}$ parameter.

The accuracy of most reported absolute magnitudes is poor due to the lack of good photometry and limited phase curve coverage. Jurić et al. (e.g. 2002) first reported a systematic error of about $0.4 \mathrm{mag}$ in the MPC's absolute magnitudes which the MPC (and others) now attempt to address with observatory-dependent corrections to the reported apparent magnitudes.

The determination of $G$ has traditionally been even more of a challenge — they are so difficult to measure that they have only been calculated for $\ll 0.1 \%$ of asteroids and, even then, the uncertainty is usually large (Pravec et al. 2012). An accurate measurement requires dense coverage of the phase curve and observations at different viewing aspects on the asteroid i.e. sub-solar positions. The vast majority of asteroids have no measured slope parameter so the average values of $G_{B}=0.15$ or $G_{M}=0.5$ are used. This assumption translates into a systematic error in an individual asteroid's $H$ and $G$, and large uncertainty on the distribution of the parameters in the population. The problem is particularly acute for objects that have been observed only at large phase angles e.g. resonant objects like 3753 Cruithne (de la Fuente Marcos and de la Fuente Marcos 2013; Wiegert et al. 1997), and objects that orbit the Sun entirely within Earth's orbit (Zavodny et al. 2008) for which absolute magnitudes might be in error by up to about 1 mag. 
In summary, the problems with our current knowledge of asteroid absolute magnitudes and slope parameters are due to:

1. Reporting observations to the Minor Planet Center (MPC) in non-standard filters and/or without accurate calibration.

2. Not performing the color transformation from the filter used for an observation to the Johnson $V$ band for an asteroid's (usually unknown) color.

3. The lack of information about the photometric uncertainty on each observation reported to the MPC so that it must be statistically 'back-calculated' for each observatory (or observer) from historical observations.

4. The MPC database storing photometric values with only 0.1 mag precision.

5. Assuming that $G_{B}=0.15$ for all asteroids that do not have a reported value for the slope parameter.

6. Sparse observations (in time). The lack of information about their rotation amplitudes induces an error and uncertainty in $H$.

7. Selection effects (Jedicke et al. 2002) that bias the discovery of asteroids towards their rotation amplitude maxima which induce a systematic error in their derived $H$.

8. Most of the effort in deriving $H$ and $G$ focuses on their statistical uncertainties when the systematic uncertainties dominate.

In this work we address each of these issues and derive the $\left(H_{B}, G_{B}\right)$ and $\left(H_{M}, G_{M}\right)$ parameters for known asteroids in the inner solar system out to, and including, Jupiter's Trojan asteroids. All the data were acquired by a single wide-field survey, Pan-STARRS1 (Kaiser et al. 2010), in standard filters with measured transformations to an accepted 
photometric system yielding photometric uncertainties that are typically about an order of magnitude smaller than earlier surveys. We use a Monte Carlo technique to measure the systematic errors introduced by filter transformations for unknown spectral types, unknown $G$, and the unknown asteroid spin and amplitude.

\section{Pan-STARRS1 asteroids.}

The Panoramic Survey Telescope and Rapid Response System's prototype telescope (Pan-STARRS1; Kaiser et al. 2010) was operated by the PS1 Science Consortium during the time period in which the data used in this study was acquired. The telescope has a 1.4 gigapixel camera (Tonry and Onaka 2009) and $1.8 \mathrm{~m} \mathrm{f} / 4$ Ritchey-Chretien optical assembly and has been surveying the sky since the second half of 2011. Although the scientific scope of the survey is wide - including the solar system, exoplanets, brown dwarfs, stellar astronomy, galaxies, cosmology, etc. - most of the data products are suitable for asteroid science. About $5 \%$ of the survey time was dedicated to the 'Solar System' (SS) survey (more accurately a survey for near-Earth objects, NEO) through the end of 2012, was increased to about 11\% from then till 2014 March 31, and the system is now $100 \%$ dedicated to NEO surveying.

Pan-STARRS1 surveys in six broadband filters, four of which were designed to be similar to the Sloan Digital Sky Survey photometric system (SDSS; Fukugita et al. 1996). Most of the observing time was devoted to the $3 \pi$ survey of the sky north of $-30^{\circ}$ declination for which each field was observed up to $20 \times /$ year in each of 5 filters $-g_{\mathrm{P} 1}, r_{\mathrm{P} 1}$, $i_{\mathrm{P} 1}, z_{\mathrm{P} 1}$ and $y_{\mathrm{P} 1}$. In the $3 \pi$ survey the same field is observed 2 or 4 times on a single night in 30-40 s exposures obtained within about an hour. The dedicated solar system survey used only the wide-band $w_{\mathrm{P} 1}$ filter that is roughly equivalent to $g_{\mathrm{P} 1}+r_{\mathrm{P} 1}+i_{\mathrm{P} 1}$ with $45 \mathrm{~s}$ exposures and a cadence of $\sim 20 \mathrm{~min}$ to image the same field $4 \times /$ night. The SS survey 
typically included fields within about $30^{\circ}$ of opposition or at small solar elongations ranging from $60^{\circ}$ to $90^{\circ}$ of the Sun.

Image processing was performed automatically and almost in real time by the Image Processing Pipeline (IPP; Magnier 2006). Transient objects were identified after 'difference imaging' (Lupton 2007) in which two consecutive images were convolved and subtracted to identify moving, or stationary but variable, targets. The photometric calibration until May 2012 was based on combined fluxes of bright stars from Tycho, USNO-B and 2MASS catalogs. Since that time the entire northern sky has been imaged by Pan-STARRS1 in all 5 filters allowing the development and use of the Pan-STARRS1 star catalog with 'ubercalibrated' magnitudes and zero points providing photometric uncertainties of $\sim 1 \%$ (Schlafly et al. 2012; Magnier et al. 2013).

Moving transient detections are identified and linked into tracklets by the Moving Object Processing System (MOPS; Denneau et al. 2013) and tracklets are associated with known asteroids by known_server (Milani et al. 2008). As of May 2015 the Pan-STARRS1 MOPS team has submitted $\sim 16,700,000$ positions and magnitudes of 575,000 known asteroids to the MPC representing $85 \%$ of all numbered asteroids. During the same time period the system discovered $\sim 41,000$ asteroids, among them about 850 NEOs and 46 comets, and reported about 2,500,000 detections of unknown asteroids to the MPC. About $\sim 42 \%$ of the detections were in the $w_{\mathrm{P} 1}$ filter acquired during the solar system survey while only about $9 \%$ were in the $y_{\mathrm{P} 1}$ and $z_{\mathrm{P} 1}$ bands.

To ensure a consistent data set of high quality photometry (Fig. 1) we restricted the detections used in this study to known asteroids in the inner solar system (out to and including Jupiter's Trojans) with multi-opposition orbits acquired during a sub-set of the $3 \pi$ and solar system surveys between February 2011 and May 2012 (see Table 1) The detections were selected from the IPP's calibrated chip-stage PSF-fit photometry (Schlafly 
et al. 2012) and were required to be unsaturated, with $\mathrm{S} / \mathrm{N}>5$, and not blended with stars or image artifacts. The Pan-STARRS1 IPP never implemented the capability of fitting trailed asteroid detections, so we restricted our data sample to asteroids that trailed by less than 5 pixels during the exposure, equivalent to the typical PSF-width of $\sim 1^{\prime \prime}$. This limited the maximum rate of motion of the asteroids to about $0.75 \mathrm{deg} / \mathrm{day}$, excluding most NEOs and even fast-moving asteroids like Hungarias and Phocaeas on the inner edge of the main belt. Our strict criteria resulted in a set of more than one million detections of approximately 240,000 asteroids

Table 1: Percentage of Pan-STARRS1 asteroid detections in each filter in the time period from February 2011 to May 2012 (values do not add to 100\% due to rounding).

\begin{tabular}{c|cccccc}
\hline Band & $g_{\mathrm{P} 1}$ & $r_{\mathrm{P} 1}$ & $i_{\mathrm{P} 1}$ & $y_{\mathrm{P} 1}$ & $z_{\mathrm{P} 1}$ & $w_{\mathrm{P} 1}$ \\
\hline Fraction (\%) & 18 & 20 & 17 & 2.2 & 6.2 & 36 \\
\hline
\end{tabular}



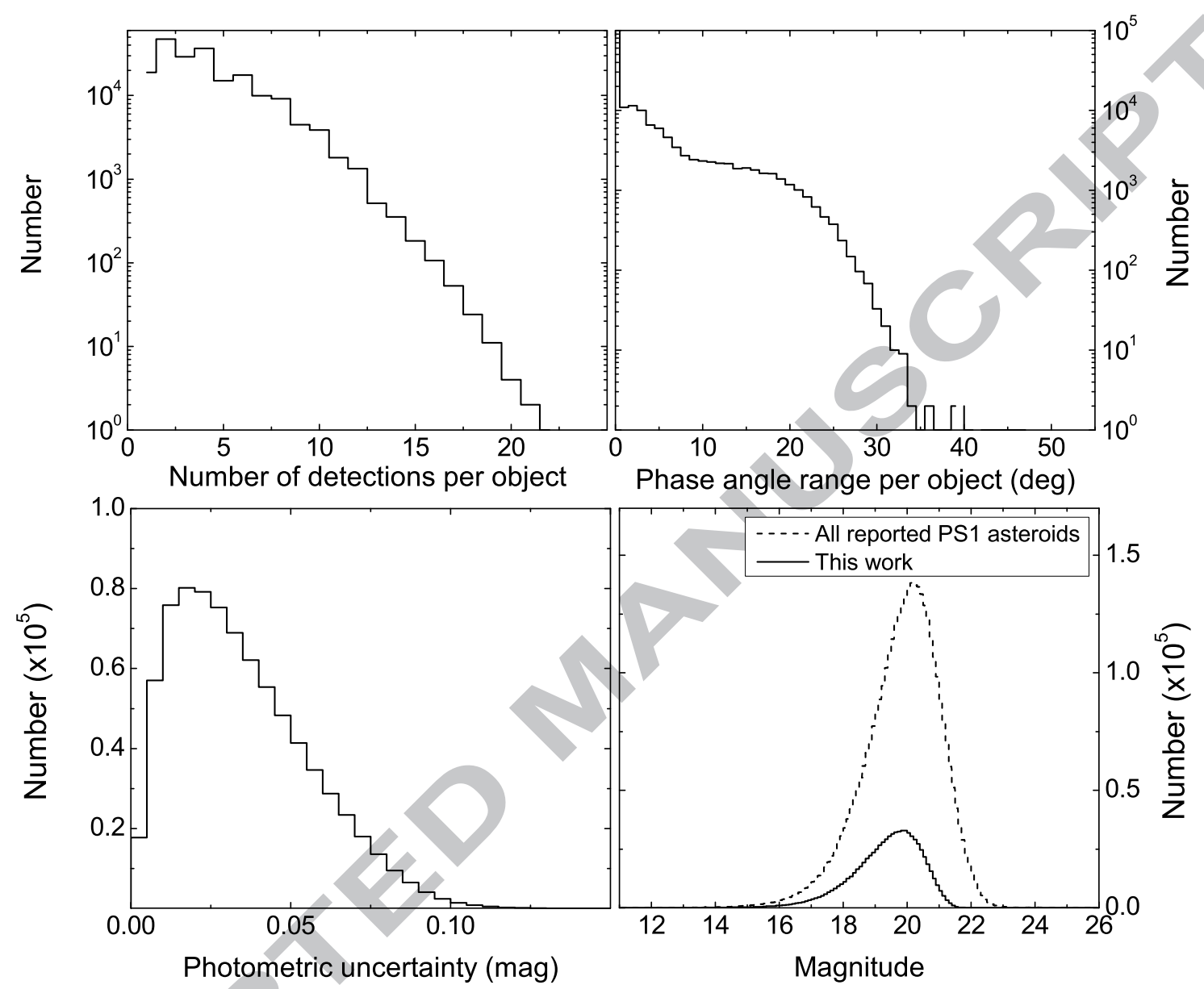

Fig. 1.- Asteroid detections used in this work. (clockwise from top left) number of detections per object, phase angle range per object, apparent $V$ magnitudes, and photometric uncertainties per detection.

Despite the enormous number of asteroid detections there are only about 10 detections/asteroid and each object is observed on average on only $\sim 3$ different nights over a phase angle range spanning about $7^{\circ}$ (Fig. 1). Therefore, the survey pattern does not typically allow the determination of an asteroid's spectral type, rotation amplitude or period. The detections have a mean \pm RMS photometric uncertainty of $0.04 \pm 0.02 \mathrm{mag}$ and 
average \pm RMS visual magnitude of $19.8 \pm 1.2 \mathrm{mag}$. The photometric uncertainty mode is $\sim 0.02$ mag corresponding to $\mathrm{S} / \mathrm{N} \sim 50$ detections. This surprisingly high value is due to our selection criteria: the multi-opposition objects were identified in earlier surveys with smaller telescopes so they are typically brighter when observed with Pan-STARRS1. Note that only $\sim 1 \%$ of the detections in our data sub-set have a photometric uncertainty greater than the 0.1 mag precision provided by the MPC.

\section{Method}

This work introduces a Monte Carlo technique to determine $H$ (and $G$ when possible) and its statistical+systematic uncertainty based on the generation of synthetic asteroids (clones) that are each consistent with the known asteroid. The power of the MC technique lies in its ability to estimate the true statistical and systematic uncertainty in the absolute magnitude due to the unknown parameters in the analysis. The clones explore the phase space of light curve rotation amplitudes, periods, colors and slope parameter in an attempt to replicate the observed apparent magnitudes. Each clone's observations are evaluated individually in the fitting process to derive $H$ and $G$ in the same manner as the actual observations so that the distribution of values for each object's clones provide a measure of the systematic errors in the values. Mathematically, our model is justified by its similarity to a sparse Bayesian marginalization over taxonomic classification, light curve amplitude and period.

\subsection{Step 1: Initial fit for $H$ and $G$}

The first step is essentially identical to the typical technique for calculating $H$ and $G$ : we fit the apparent $V$-band magnitude to the B89 and M10 phase functions using 
the IDL procedure mpfit2dfun ${ }^{1}$ that employs the Levenberg-Marquardt least-squares fitting technique (Levenberg 1944; Marquardt 1963) to minimize the variance between the detections' apparent magnitudes and the values predicted by the models. We converted the Pan-STARRS1 apparent magnitudes to $V$-band using taxonomy-dependent filter transformations if the asteroid's taxonomy was specified by Hasselmann et al. (2012) and, if not, the mean $\mathrm{S}+\mathrm{C}$ class color (see Table 2).

Table 2: Asteroid magnitude transformations from Pan-STARRS1 AB filter magnitudes to the Johnson-Cousin $V$ system based on Tonry et al. (2012). Solar colors are also included for reference.

\begin{tabular}{c|cccccc}
\hline \hline Taxonomy & $\mathrm{V}-g_{\mathrm{P} 1}$ & $\mathrm{~V}-r_{\mathrm{P} 1}$ & $\mathrm{~V}-i_{\mathrm{P} 1}$ & $\mathrm{~V}-z_{\mathrm{P} 1}$ & $\mathrm{~V}-y_{\mathrm{P} 1}$ & $\mathrm{~V}-w_{\mathrm{P} 1}$ \\
\hline Sun & -0.217 & 0.183 & 0.293 & 0.311 & 0.311 & 0.114 \\
$\mathrm{Q}$ & -0.312 & 0.252 & 0.379 & 0.238 & 0.158 & 0.156 \\
$\mathrm{~S}$ & -0.325 & 0.275 & 0.470 & 0.416 & 0.411 & 0.199 \\
$\mathrm{C}$ & -0.238 & 0.194 & 0.308 & 0.320 & 0.316 & 0.120 \\
$\mathrm{D}$ & -0.281 & 0.246 & 0.460 & 0.551 & 0.627 & 0.191 \\
X & -0.247 & 0.207 & 0.367 & 0.419 & 0.450 & 0.146 \\
\hline Mean (S+C) & -0.28 & 0.23 & 0.39 & 0.37 & 0.36 & 0.16 \\
\hline \hline
\end{tabular}

The initial fits also use the mean class-dependent $G$ provided in Table 3 if the taxonomic class is specified in the SDSS database (Hasselmann et al. 2012) but, if the class is not known, we use the mean of the S- and C- class values: $\bar{G}_{B}=0.15$ (B89) and $\bar{G}_{M}=0.53$ (Oszkiewicz et al. 2012) respectively.

The initial fits provided the absolute magnitudes in both photometric systems, $H_{B, i}$

\footnotetext{
${ }^{1}$ Markwardt IDL library, http://www.physics.wisc.edu/ craigm/idl
} 
Table 3: Average slope parameters, $G_{B}$ and $G_{M} \equiv G_{12}$, adopted in this work for 5 asteroid taxonomic classes as measured by Pravec et al. (2012) and Oszkiewicz et al. (2012) respectively. The 6th row provides 'standard' averages for the dominant $\mathrm{S}$ and $\mathrm{C}$ taxonomies.

\begin{tabular}{|c|c|c|}
\hline \hline $\begin{array}{c}\text { Taxonomic } \\
\text { Class }\end{array}$ & $\begin{array}{c}G \equiv G_{B} \\
\pm(\mathrm{RMS})\end{array}$ & $\begin{array}{c}G_{12} \equiv G_{M} \\
\pm(\mathrm{RMS})\end{array}$ \\
\hline $\mathrm{Q}$ & $0.25 \pm 0.13$ & $0.41 \pm 0.14$ \\
$\mathrm{~S}$ & $0.24 \pm 0.06$ & $0.41 \pm 0.16$ \\
$\mathrm{C}$ & $0.15 \pm 0.09$ & $0.64 \pm 0.16$ \\
$\mathrm{D}$ & $0.09 \pm 0.09$ & $0.47 \pm 0.14$ \\
$\mathrm{X}$ & $0.20 \pm 0.09$ & $0.48 \pm 0.19$ \\
\hline $\mathrm{S}+\mathrm{C}$ & 0.15 & 0.53 \\
\hline \hline
\end{tabular}

and $H_{M, i}$, that were the inputs to the next step in the pipeline.

\subsection{Step 2: Generating asteroid clones}

Our final $H$ and $G$ estimates are the result of Monte Carlo (MC) simulations that require the generation of synthetic 'clones' for each of the asteroids in our sample. Each of the clones is generated with its own color, slope parameter, rotation period, light curve amplitude and phase, where each of the parameters is selected from a unbiased distribution as described below. 


\subsubsection{Clone colors}

Our pipeline can assign each clone the color of its parent asteroid (if known) or, when the parent's color is not known, a random color based on an appropriate mix of taxonomies as a function of semi-major axis. About $16 \%$ of the asteroids in our sample have taxonomies defined by Hasselmann et al. (2012) (SDSS).

We implemented this technique by dividing the inner solar system into 4 zones (see table 4): NEO-like $(a<2 \mathrm{au})$, main belt ( 2 au $\leq a<3.7 \mathrm{au}$ ), Hildas $(3.7 \mathrm{au} \leq a<4.5 \mathrm{au})$ and Trojans $(4.5 \mathrm{au} \leq a<6.0 \mathrm{au})$. The semi-major limits defining the zone edges were set at or near a minimum in the number distribution as a function of semi-major axis and by the availability of published taxonomic distributions. The exact values make little difference to this work. We used the published, debiased taxonomic distributions in Table 4 in the 4 zones with the qualification that for the main belt (Mothé-Diniz et al. 2003) we aggregated many related taxonomic types into 3 broad spectral classes: $\mathrm{S}$-class $=(\mathrm{A}, \mathrm{AQ}, \mathrm{AV}, \mathrm{O}, \mathrm{OV}$, S, SA, SO, SQ, SV, V, L, LA, LQ, LS), X-class=(X, XD, XL, XS), and Q-class=(Q, QO, QV). We required that the fraction, $f(c, z)$, of asteroids with spectral class $c$ in zone $z$ satisfies $\sum_{c} f(c, z)=1$. In the main belt, zone 2 , we were able to generate the taxonomies as a finer function of $a$ as provided by (Mothé-Diniz et al. 2003) with a similar requirement that $\sum_{c} f(c, a)=1$ at each semi-major axis.

\subsubsection{Clone slope parameters}

We assigned slope parameters to the clones as a function of their assigned taxonomic class $(c)$. i.e. the $k^{t h}$ clone was assigned a slope parameter $G_{k}(c)=\operatorname{ran}\left[\overline{\mathrm{G}}(\mathrm{c}), \sigma_{\mathrm{G}}(\mathrm{c})\right]$ where $\operatorname{ran}[\mathrm{x}, \mathrm{y}]$ is a random number generated from a normal distribution with mean $x$ and standard deviation $y$, and $\bar{G}(c)$ and $\sigma_{G}(T)$ are the mean and RMS of the distribution of 
Table 4: Taxonomic distribution of asteroids in 4 semi-major axes zones used in this work. The main belt values are given below at a representative $a=2.5 \mathrm{au}$ but we generated the clone taxonomies as a smooth function of semi-major axis in the range $2.0 \mathrm{au} \leq a<3.2$ au as specified by Mothé-Diniz et al. (2003).

\begin{tabular}{c|cccc}
\hline \hline & zone 1 & zone 2 & zone 3 & zone 4 \\
Taxonomy & NEO-like $^{\mathrm{a}}$ & $\mathrm{MB}^{\mathrm{b}}$ & Hilda $^{\mathrm{c}}$ & Trojans $^{\mathrm{d}}$ \\
& $a<2 \mathrm{au}$ & $a \sim 2.5 \mathrm{au}$ & $3.7 \mathrm{au} \leq a<4.5 \mathrm{au}$ & $4.5 \mathrm{au} \leq a<6 \mathrm{au}$ \\
$\mathrm{Q}$ & 14 & 0 & 0 & 0 \\
$\mathrm{~S}$ & 23 & 61 & 0 & 0 \\
$\mathrm{C}$ & 10 & 30 & 7 & 10 \\
$\mathrm{D}$ & 18 & 0 & 67 & 80 \\
$\mathrm{X}$ & 35 & 9 & 26 & 10 \\
\hline \hline
\end{tabular}

${ }^{a}$ Stuart and Binzel (2004)

b Mothé-Diniz et al. (2003)

c Grav et al. (2012)

d Grav et al. (2012)

slope parameters for class $c$, respectively (Table 3).

\subsubsection{Clone rotation periods, amplitudes and phases}

The sparse Pan-STARRS1 data did not allow us to measure any asteroid's rotation period and light curve amplitude. Furthermore, $\lesssim 2 \%$ of the asteroids in our sample have 
measured light curves reported in the asteroid light curve database (LCDB ${ }^{2}$; Warner et al. 2009; Waszczak et al. 2015) The lack of this information introduces systematic uncertainty and error into the absolute magnitude and slope parameter determination. We quantified these effects using our Monte Carlo technique with synthetic sinusoidal light curves for each clone.

Asteroid brightness variations on the hours-to-days timescales are usually caused by their non-spherical shape and rotation (the exceptions are for the unusual cases where the phase angle changes rapidly for close approaching NEOs, for multiple-systems in which brightness changes can occur if the objects transit or eclipse each other, and for objects with significant color variations). We assumed that the observing geometry (i.e. phase angle) effect on the asteroids' light curves are negligible in the Pan-STARRS1 data because of the limited range in phase angle coverage in our sample (Fig. 1). For the purpose of generating the clones' light curves we assumed that all objects generate simple sinusoidal light curves with peak-to-peak amplitude $A$, period $P$, and rotation phase $\theta$. The offset from the unmodulated light curve at time $t$ is then $\Delta m(t)=A \sin (2 \pi t / P+\theta) / 2$.

Light curve amplitudes tend to be larger for smaller asteroids (see Fig. 2, Warner et al. 2009), probably because the smaller objects tend to be more irregularly shaped. Overall, the set of measured amplitudes and periods will be larger and shorter respectively than the true distribution because of observational selection effects, larger amplitudes and shorter periods are easier to detect and measure.

To reduce the impact of the light curve amplitude and period selection effects we employed the debiased distributions derived by Masiero et al. (2009) that are representative

${ }^{2}$ The asteroid lightcurve database is publicly available at http://www.minorplanet.info/lightcurvedatabase.html 

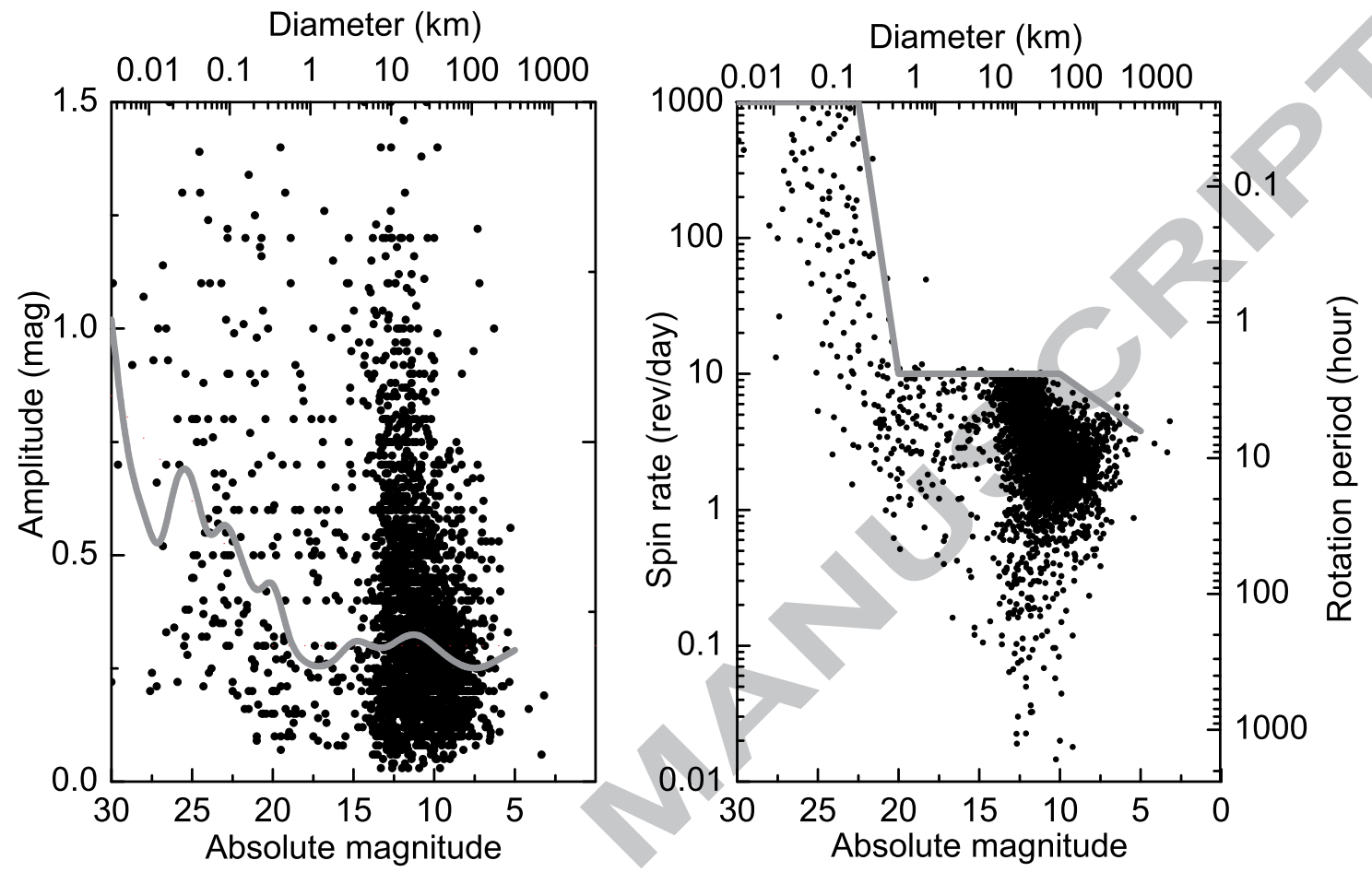

Fig. 2.- (left) Asteroid light curve amplitudes vs. absolute magnitude $\left(H_{B}\right)$ from the LCDB (Warner et al. 2009). The solid gray curve represents the size-dependent moving median in 1.0 mag wide bins. (right) Measured asteroid spin rates (periods are provided on the right) vs. absolute magnitude $\left(H_{B}\right)$ from the LCDB (Warner et al. 2009). The solid gray curve represents the size-dependent upper strength limit derived by Holsapple (2007).

of asteroids with $H \sim 18$ (the average \pm RMS absolute magnitude in their study was $17.7 \pm 1.4 \mathrm{mag})$. i.e. for objects with $H \sim 18$ they provide the cumulative fraction of asteroids, $F_{a m p, M a s}(A)$, with light curve amplitudes $<A$. We empirically estimate the cumulative distribution of light curve amplitudes at other absolute magnitudes $F_{a m p}(A, H)$ 
by 'normalizing' to the median at $H=18$ from the median at other values:

$$
F_{\text {amp }}(A, H)=F_{\text {amp }, \text { Mas }}\left[A \times \frac{A_{\text {med }}(18)}{A_{\text {med }}(H)}\right]
$$

where $A_{\text {med }}(H)$ is an empirical function (Fig. 2) representing the median amplitude of asteroids in the LCDB (Warner et al. 2009). Thus, given a clone's initial (§3.1) absolute magnitude, $H_{i}$, we generated a random light curve amplitude for the clone according to the cumulative fractional distribution given by eq. 2 .

We followed a similar procedure in assigning each clone a rotation rate $R$ or, equivalently, a rotation period $P \equiv 1 / R$. Masiero et al. (2009) also provide the data from which we derive the cumulative fraction of asteroids, $F_{\text {rot, Mas }}(R)$, with rotation rates $<R$. Once again, their results are representative of asteroids with $H \sim 18$, about 2 mag fainter than the mean value in our data sample, so we developed an empirical technique to extend their cumulative fractional rotation rate distribution to other absolute magnitudes.

Asteroids with diameters $\gtrsim 100 \mathrm{~m}(H \lesssim 23)$ have an empirically observed upper limit to their rotation rate of about $12 \mathrm{rev/day} \mathrm{(Fig.} \mathrm{2)} \mathrm{and} \mathrm{about} 99 \%$ of the distribution of debiased spin rates are $<12 \mathrm{rev} /$ day (Masiero et al. 2009). Asteroids larger than a few tens of kilometers $(H \lesssim 12)$ have an even more restricted upper limit to their rotation rates. We empirically defined an $R_{\max }(H)$ as illustrated in fig. 2 and 'compress' or 'expand' the Masiero et al. (2009) distribution as necessary to create the cumulative fractional distribution at any $H$ :

$$
F_{\text {rot }}(R, H)=F_{\text {rot }, \text { Mas }}\left[R \times \frac{R_{\max }(18)}{R_{\max }(H)}\right] .
$$

Once again, given a clone's initial (§3.1) absolute magnitude, $H_{i}$, we generated a random rotation rate for the clone according to the cumulative fractional distribution given by eq. 3 .

Finally, the rotational phase $\theta_{k}$ for the $k^{\text {th }}$ clone was generated from a random uniform distribution in the range $\left[0^{\circ}, 360^{\circ}\right)$. 
Our light curves were simple sinusoids even though we understand that real asteroid light curves can be much more complicated. The technique could easily be extended to incorporate actual light curve properties or a more realistic distribution but i) only a tiny fraction of known asteroids have measured light curves ii) we will show below that our results are not particularly sensitive to the actual light curve parameter distribution and iii) if the actual light curve is known then there is no need for any of the methods developed here. i.e. this method only applies to the $98 \%$ of asteroids that do not have measured light curves. Since this is a preliminary work we have not made any effort to remove those asteroids that have published light curves.

\subsection{Step 3: Refining $H$ and $G$ (First Monte Carlo simulation).}

The first Monte Carlo (MC) simulation yields our MC estimate for $H$ and $G$ from the sparse Pan-STARRS1 phase curve coverage data. As described in detail above, we created 500 clones of each object where the $k^{t h}$ clone was assigned a taxonomic class (color) $c_{k}$, light curve amplitude $A_{k}$, and period $P_{k}$. We then fit for each clone's absolute magnitude, slope parameter and light curve phase, $\left(H_{k}^{\prime}, G_{k}^{\prime}, \theta_{k}^{\prime}\right)$, by minimizing the $\chi^{2}$ with respect to the actual observations:

$$
\chi_{k, o b s}^{2}=\sum_{j=1}^{n}\left[\frac{m_{k}\left(t_{j} ; H_{k}^{\prime}, G_{k}^{\prime}, \theta_{k}^{\prime}\right)-m\left(t_{j}\right)}{\delta m\left(t_{j}\right)}\right]^{2}
$$

where $n$ is the number of observations (detections) of the object, $m\left(t_{j}\right)$ is the actual object's observed apparent magnitude, $\delta m\left(t_{j}\right)$ is the reported uncertainty on the actual Pan-STARRS1 apparent magnitude for that observation in the original filter, and $m_{k}$ is the clone's predicted apparent magnitude at the actual time of observation, $t_{j}$, in the Pan-STARRS1 filter in which the observation was made, with the clone's appropriate color 
transformation $\left(\Delta m_{k}\left(t_{j}\right)\right.$; Table 2$)$ :

$$
m_{k}\left(t_{j}\right)=5 \log \left[r\left(t_{j}\right) \Delta\left(t_{j}\right)\right]+\Phi\left[\alpha\left(t_{j}\right) ; H_{k}^{\prime}, G_{k}^{\prime}\right]+A_{k} \sin \left[2 \pi t_{j} / P_{k}+\theta_{k}^{\prime}\right] / 2+\Delta m_{k}\left(t_{j}\right)
$$

and $\Phi$ is the B89 or M10 phase function.

The 'best' clone is the one $\left(k^{*}\right)$ that produces the minimum $\chi^{2}$ and we adopt that clone's $H_{k^{*}}^{\prime}$ and $G_{k^{*}}^{\prime}$ values as our MC estimate for the object's absolute magnitude and slope parameter. The process was run separately for both the B89 and M10 phase functions to provide our MC estimates for $\left(H_{B}, G_{B}\right)$ and $\left(H_{M}, G_{M}\right)$ respectively. To avoid unphysical values the fitting process required that $-0.25 \leq G_{B} \leq 0.8$ and $-0.5 \leq G_{M} \leq 1.5$.

We found that 500 clones provides a good balance between the computation time and our ability to estimate the uncertainty on the absolute magnitudes and slope parameters. It is likely that when there are only a small number of detections that the number of clones could be decreased but we did not pursue this simplification. When the number of detections becomes very large then our technique becomes unnecessary as either traditional (Pravec et al. 2012) or sparse light curve fitting (Muinonen et al. 2010; Law et al. 2009) becomes more effective.

\subsection{Step 4: Estimating uncertainties and error on $H$ and $G$ (second Monte Carlo fit).}

At the risk of being pedantic, error is the difference between the value of a measurement and the true value of the measurand, and uncertainty is an estimate of the error applicable to a measurement. We estimated the uncertainties and errors on $H_{k^{*}}^{\prime}$ and $G_{k^{*}}^{\prime}$ by fitting for the absolute magnitude and slope parameter with purely synthetic light curves generated from the clone with the best fit. i.e. we re-applied the same method as described in Step 3 (§3.3) except that we fit the clones to the best synthetic object rather than the real object 
(we continue to use the sub-script $k$ to refer to clones but the clones used here are distinct from the clones used in the last step):

$$
\chi_{k, \text { syn }}^{2}=\sum_{i=1}^{n}\left[\frac{m_{k}\left(t_{j} ; H_{k}^{\prime}, G_{k}^{\prime}, \theta_{k}^{\prime}\right)-m_{k^{*}}\left(t_{j}\right)}{\delta m_{k^{*}}\left(t_{j}\right)}\right]^{2} .
$$

where $\delta m_{k^{*}}\left(t_{j}\right)=\delta m\left(t_{j}\right)$, i.e. the uncertainty on the synthetic observation at time $t_{j}$ was set to the uncertainty on the actual observation at time $t_{j}$.

If we let $X$ generically represent either $H$ or $G$ then the combined statistical+systematic uncertainty (later denoted as uncertainty) on $X$ is the standard deviation of the clones' $X$ distribution:

$$
\delta X=\sqrt{\frac{1}{n} \sum_{k}\left(X_{k}^{\prime}-\overline{X^{\prime}}\right)^{2}}
$$

where $\overline{X^{\prime}}$ is the average value of $X$ for all the synthetic objects' clones. Similarly, the combined statistical+systematic error (later denoted as error) on $X$ is the average error on the values for the synthetic clones:

$$
\Delta X=\frac{1}{n} \sum_{k}\left(X_{k}^{\prime}-X_{k^{*}}^{\prime}\right)
$$

\subsection{Verification}

We verified our method with two independent sets of synthetic data generated from real Pan-STARRS1 data: 1) 10,000 randomly selected known Pan-STARRS1 objects, most of them with sparse phase curve coverage and 2) the 1,000 known Pan-STARRS1 objects with the best phase coverage. To have better control over assessing our method's validity we generated photometric magnitudes and uncertainties with synthetic absolute magnitudes $\left(H_{B}\right.$ and $\left.H_{M}\right)$ and slope parameters $\left(G_{B}\right.$ and $\left.G_{M}\right)$ at each real time of observation with the known object's orbit. We then employed our pipeline to calculate each synthetic object's $H$ and $G$ to measure the statistical and systematic errors induced by our technique. Moreover, 
we tested two different scenarios for assigning light curve amplitudes and periods to the clones: 1) the debiased distributions from Masiero et al. (2009), 2) and the observed distributions from the LCDB (Warner et al. 2009).

The result is that for both synthetic populations (sparse and dense phase curve coverage) and for both light curve amplitude-period relations (debiased and observed) the difference between the generated synthetic values and the values returned by our method was normally distributed with zero mean. i.e. our technique correctly derives the $H$ and $G$. Use of the debiased or observed amplitude and period distributions does not affect the derived $H$ and $G$ at the level of photometric accuracy and uncertainty of the Pan-STARRS1 data with its associated phase curve coverage i.e. does not cause any systematic errors.

\section{Results \& Discussion}

\subsection{Absolute magnitudes comparison with Pravec et al. (2012)}

We think Pravec et al. (2012)'s detailed light curve study of $\sim 500$ asteroids sets the standard in measuring asteroid photometric properties. They provided only $H_{B}$ (it was before the adoption of the new IAU standard) but that value should be identical to $H_{M}$. Our results agree with Pravec et al. (2012) for the 347 objects that appear in both data sets (fig. 3). The mean differences of $H_{B}-H_{B, P r a}=-0.06 \pm 0.02 \mathrm{mag}$ and

$H_{M}-H_{B, P r a}=0.02 \pm 0.02 \mathrm{mag}$ are consistent with zero to within $3 \sigma$ and $1 \sigma$ respectively, with better agreement for the new IAU standard photometric system of M10. The RMS of each distribution is $0.36 \mathrm{mag}$ and $0.29 \mathrm{mag}$ respectively, due to the quadratic combination of the errors in both Pravec et al. (2012)'s and this work.

The distribution of $H_{B}-H_{B, P r a}$ is quasi-normally distributed (fig. 3) with an RMS of 0.31 mag including a tail extending to $H_{B}-H_{B, P r a}<-1$. Interestingly, the difference 

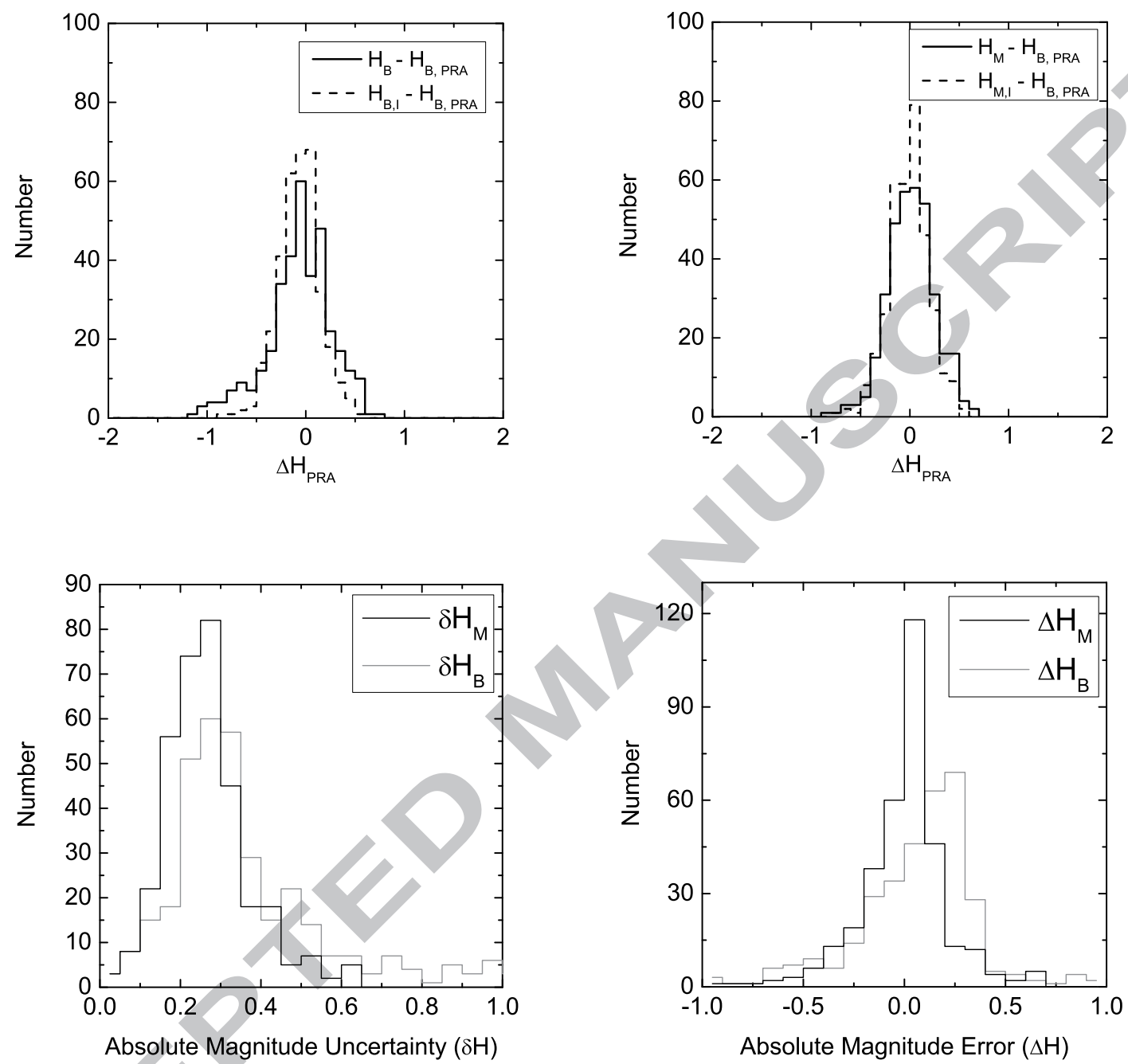

Fig. 3.- (top) Absolute magnitudes from our study compared with 347 objects in common with Pravec et al. (2012) using the B89 (left) and M10 (right) photometric systems. The dashed line shows the results of the traditional initial fit (§3.1) and the solid line provides the results of the MC fit (§3.3). (bottom-left) Uncertainties and (bottom-right) estimated systematic errors on absolute magnitudes from our study compared with those reported by Pravec et al. (2012). 
between our initial fits with assumed slope parameter (§3.1) and Pravec et al. (2012), $H_{B, i}-H_{B, P r a}$, is roughly normally distributed with a mean error of $-0.06 \pm 0.02$ mag and RMS of 0.26 mag. Thus, the simple, traditional, fitting procedure with assumed $G$ to our high-precision but sparse data produces comparable absolute magnitudes to the MC technique.

Our absolute magnitudes calculated with the M10 phase function $\left(H_{M}\right)$ are better behaved (fig. 3) in the sense that the distribution is more normally distributed. The initial fit to the sparse data in the M10 system provided absolute magnitudes with mean systematic errors of $0.00 \pm 0.02 \mathrm{mag}$ and $\sigma \sim 0.26 \mathrm{mag}$ compared to the MC technique with a mean error of $0.02 \pm 0.02 \mathrm{mag}$ and $\sigma \sim 0.28 \mathrm{mag}$. The good behavior of both the MC and initial fits with M10 that results in a normal error distribution leads us to the conclusion that it is superior for the determination of absolute magnitudes even for sparse data samples.

We also used the Pravec et al. (2012) values to test our technique (§3.4) for establishing the uncertainty and error on our measured absolute magnitudes. Their technique allows excellent control of all the statistical and systematic uncertainties in the $H$ calculation because they observed targets for more than a decade in a systematically controlled program and had 2 to 3 orders of magnitude more data per object. Thus, they report $H$ uncertainties about $3 \times$ less than our uncertainties and we can compare our measured uncertainties $(\delta H)$ to the RMS spread of $H-H_{P r a}$, and our measured error estimates to its average (fig. 3).

As stated earlier, the real power of the MC technique is its ability to estimate the statistical and systematic uncertainties on the derived $H$ and $G$ values. Our estimated absolute magnitude uncertainties $\left(\delta H_{B}\right.$; fig. 3 ; §3.4) for the asteroids that overlap the Pravec et al. (2012) data sample have the expected poissonian distribution with a mean of $\bar{\delta}_{H_{B}}=0.36 \pm 0.01 \mathrm{mag}$ using the B89 phase function (fig. 3), comparable to the RMS 
of $0.37 \pm 0.02 \mathrm{mag}$ for the distribution of the error in our measurement, $H_{B}-H_{B, \text { Pra }}$, as expected. Similarly, our mean estimated systematic error of $\Delta H_{B}=0.03 \pm 0.02 \mathrm{mag}$ agrees with the actual systematic offset in the $H_{B}-H_{B \text {,Pra }}$ distribution. We can compare our estimated uncertainties and systematic errors in the same manner for the M10 phase curve. Our estimated mean uncertainty, $\delta H_{M}=0.26 \pm 0.01 \mathrm{mag}$, is consistent with $R M S\left(H_{M}-H_{B, \operatorname{Pra}}\right)=0.28 \pm 0.02 \mathrm{mag}$ and our estimated systematic error of $\Delta H_{M}=0.00 \pm 0.02 \mathrm{mag}$, is consistent with $\overline{\left(H_{M}-H_{B, P r a}\right)}=0.02 \pm 0.02 \mathrm{mag}$.

The good agreement between our results and those of Pravec et al. (2012) illustrates the utility of our MC technique at measuring an asteroid's absolute magnitude and estimating the associated statistical+systematic uncertainty and any systematic bias, even for sparse data sets with limited phase angle coverage. Furthermore, the nice behavior of our results with the M10 phase curve and the good agreement between our $H_{M}$ and $H_{B, \text { Pra }}$ provides evidence that $H_{M} \sim H_{B}$ when care is taken to ensure that the photometric data is of excellent quality.

\subsection{Absolute magnitudes}

Having established the utility of our technique on a well-controlled data set in the previous section we now employ it on all the asteroids in our selected Pan-STARRS1 data sample. We were able to calculate the absolute magnitudes with combined statistical and systematic uncertainties for more than 240,000 asteroids spanning the range from $6.4<H<26.5$ (fig. 4). Our results include objects ranging from NEOs in the inner solar system to Jupiter's Trojan asteroids. Our sample represents $\sim 38 \%$ of all known asteroids in that range as of February 2014, with the highest completion of $\sim 75 \%$ from $10.5<H<11.0$. 

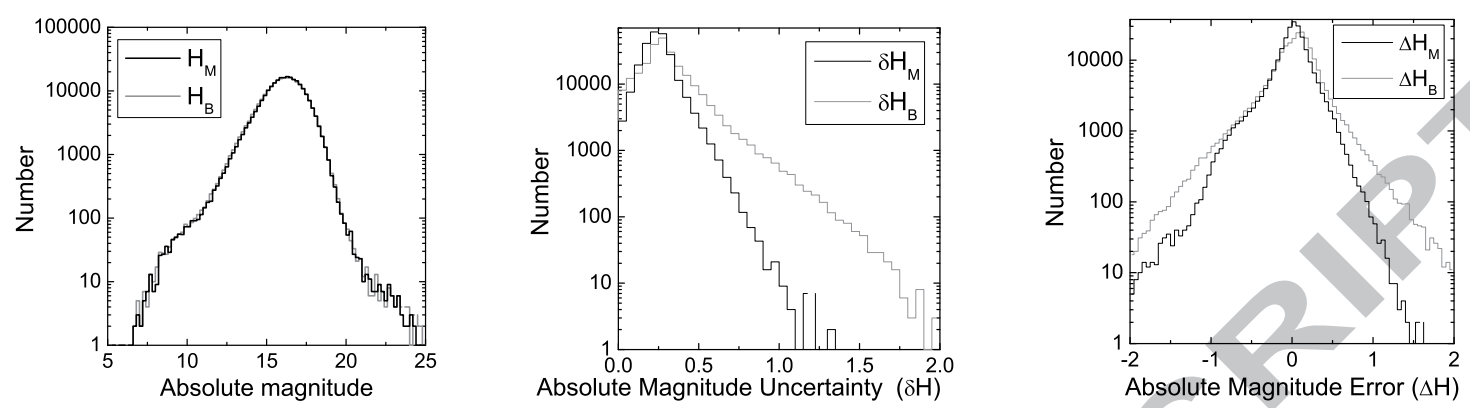

Fig. 4. - (left) Absolute magnitudes $\left(H_{M}\right.$ and $\left.H_{B}\right)$ of 248,457 asteroids. (center) Uncertainties and (right) estimated errors in the absolute magnitudes derived with our Monte Carlo method using the phase functions of (gray) B89 and (solid) M10.

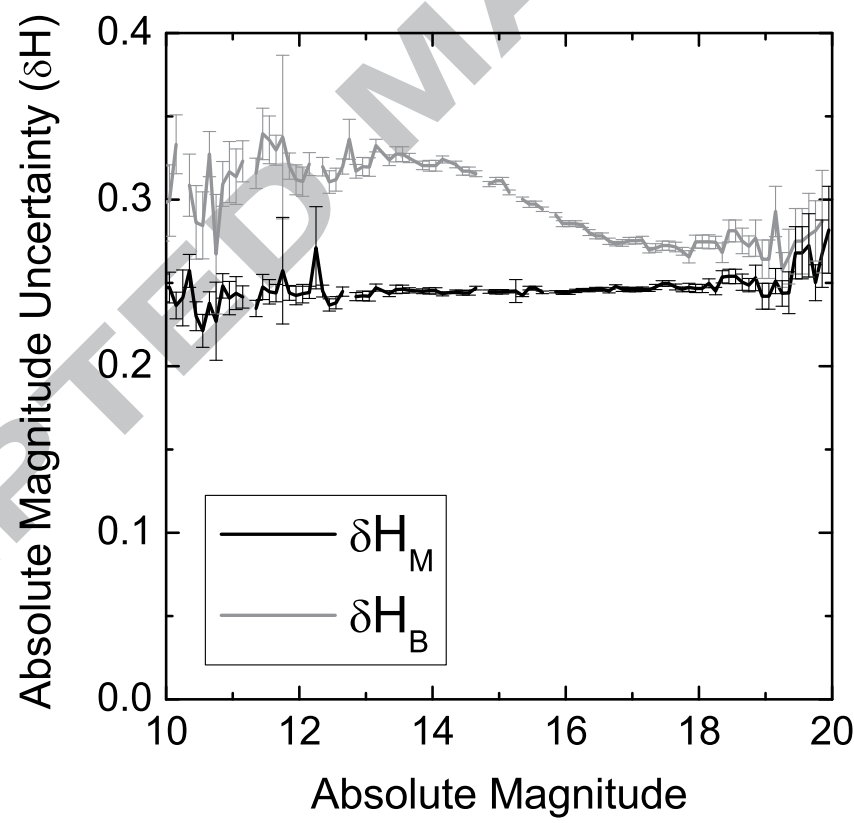

Fig. 5.- Absolute magnitude uncertainty as a function of $\mathrm{H}$ using the B89 (grey) and M10 (dark) methods. 
The mean uncertainties of $\overline{\delta H_{B}}=0.30 \pm 0.01 \mathrm{mag}$ and $\overline{\delta H_{M}}=0.25 \pm 0.01 \mathrm{mag}$ (Fig 4) show that the new IAU photometric scheme of M10 is better than B89 for the sparse Pan-STARRS1 data and phase coverage but this conclusion mis-represents the full utility of the M10 technique. For one, the M10 system uncertainty is almost uniform with $\delta H_{M} \sim 0.24 \mathrm{mag}$ for the entire range $10<H<20$ (fig. 5). Second, even though the two techniques yield approximately the same uncertainties for the faintest objects for which the uncertainty is dominated by the measurement statistics (fig. 5), the B89 method's statistical uncertainty is $\sim 0.35 \%$ larger for bright objects $(10<H<14)$.

The mean of our estimated statistical+systematic error using the M10 method, $\overline{\left|\Delta H_{M}\right|}=0.02 \pm 0.01 \mathrm{mag}$, is comparable to the B89 method, $\overline{\left|\Delta H_{B}\right|}=0.01 \pm 0.01 \mathrm{mag}$ (fig. 4). The error in the absolute magnitude for each asteroid is less than the estimated uncertainty in $\sim 62 \%$ of all the asteroids in our $H_{B}$ sample and $\sim 73 \%$ in our $H_{M}$ sample. The RMS of the $\left|\Delta H_{B}\right|$ and $\left|\Delta H_{M}\right|$ errors respectively of $\sim 0.35 \mathrm{mag}$ and $\sim 0.25 \mathrm{mag}$ confirms that the new IAU photometric system is an improvement over the earlier one and, furthermore, the shape of the error distribution is more reasonable for $\Delta H_{M}$ than $\Delta H_{B}$ (note the peak of $\Delta H_{B}$ is shifted by $0.05 \mathrm{mag}$ from zero but the $\Delta H_{M}$ peak is near zero (fig. 4).

Overall, there is almost no difference between our M10 and B89 ensemble results for Pan-STARRS1 asteroids and the mean difference $\overline{H_{M}-H_{B}}$ is $0.03 \pm 0.01 \mathrm{mag}$ with RMS of $0.22 \mathrm{mag}$ (fig. 6). The mean difference between the initial fit solutions is $\overline{H_{M, i}-H_{B, i}}=0.05 \pm 0.01 \mathrm{mag}$.

On the other hand, the utility of restricting $H$ and $G$ analyses to data derived from well-calibrated single-survey data is easily illustrated by comparing the results of our technique to the MPC database values that do their best to incorporate data from multiple telescopes and observers over many decades. The MPC currently only publishes 

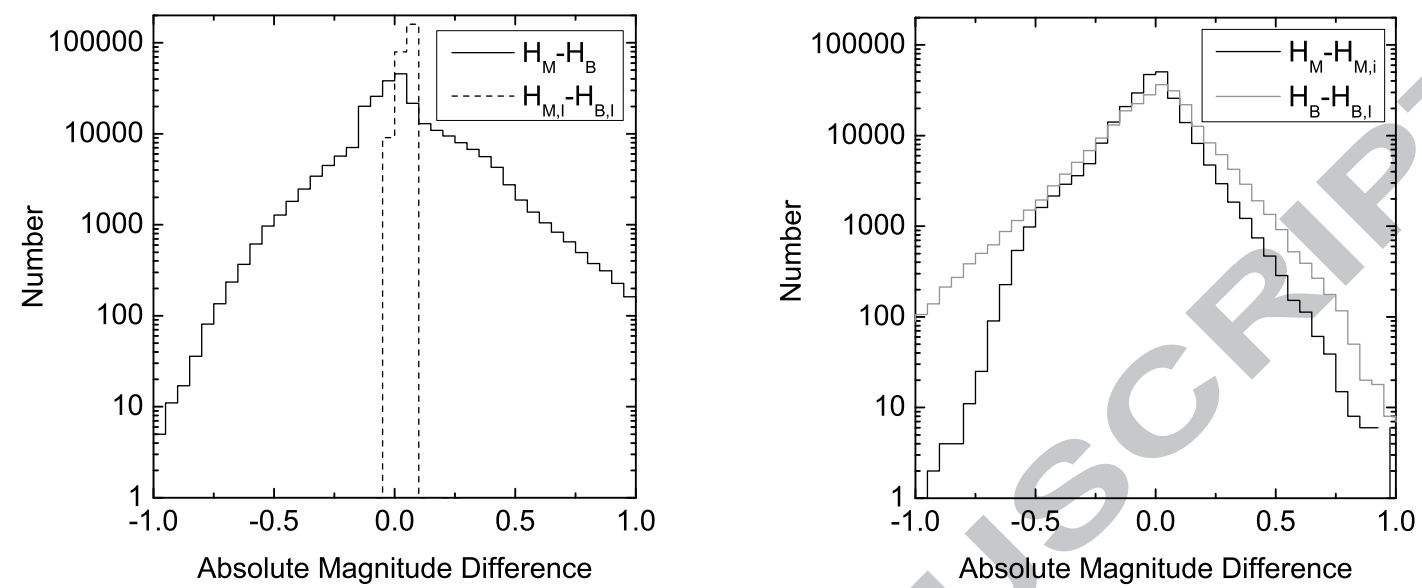

Fig. 6. - (left) Difference between the M10 and B89 absolute magnitudes for the MC and initial fit solutions. (right) Difference between MC and initial fit solutions for the absolute magnitude using the M10 and B89 methods.

absolute magnitudes using the B 89 phase function and there is a mean difference of $\overline{H_{M}-H_{B, M P C}}=0.26 \pm 0.01 \mathrm{mag}$ and $\overline{H_{B}-H_{B, M P C}}=0.22 \pm 0.01 \mathrm{mag}$ between our technique and the MPC values. The consistency between the mean differences is at least reassuring and the RMS spread in values is due to 1) the systematics introduced by the MPC's procedure that incorporates apparent magnitudes from many different observatories in many different passbands and 2) the systematics introduced by our sparse light curve coverage. Given that we established in $§ 4.1$ that our technique works well in comparison to the 'standard' Pravec et al. (2012) values, our conclusion is that the error is due to the MPC's incorporation of photometry from different sites and filters over a long period of time. The error reported here is less than the $\sim 0.4$ mag value reported by Juric et al. (2002), but since the time of that study the MPC database has been further populated by photometry from Pan-STARRS1 and other large surveys with better photometric calibrations than previous surveys. Hence, it is unsurprising that the $H_{B, M P C}$ values 

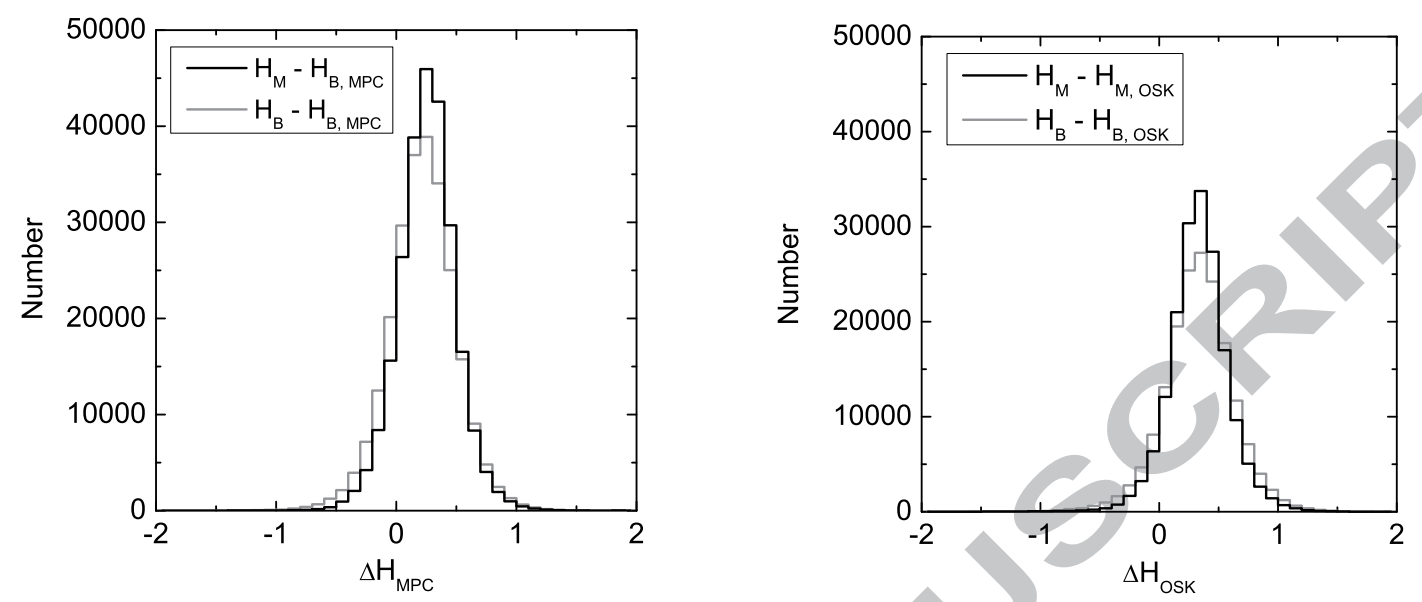

Fig. 7.- The difference between the $H_{B}$ and $H_{M}$ absolute magnitudes calculated in this work and (left) the Minor Planet Center and (right) Oszkiewicz et al. (2012). We compare our $H_{M}$ to the MPC $H_{B}$ because the two photometric systems should yield similar absolute magnitudes (in theory).

approach their correct values over time.

Our calculated uncertainties are about $2 \times$ larger than reported by Oszkiewicz et al. (2012) who employed the entire MPC catalog for their input photometry and provided $H_{M}$ and $G_{M}$ for 421,496 asteroids - almost double our sample. For comparison with earlier works they also provided $H_{B}$ and $G_{B}$. Their work was very difficult as it required calibrating and correcting the systematic problems intrinsic to the various observatories and observers that contributed the photometric data in multiple filters, but offered the advantage of an extensive data set with wide time and phase angle coverage i.e. much like the MPC technique described in the last paragraph. The systematic offset between our $H_{M}$ values and Oszkiewicz et al. (2012) of $H_{M}-H_{M, O s k}=0.33 \pm 0.01 \mathrm{mag}$ (fig. 7) is similar to the offset derived between our results and the MPC.

Jurić et al. (2002) and Pravec et al. (2012) reported a systematic offset of about 
$0.38 \mathrm{mag}$ to $0.5 \mathrm{mag}$ between their calculated absolute magnitudes and the values reported by the MPC while Parker et al. (2008) found an $\sim 0.33$ mag offset between their $H_{B}$ and the $\mathrm{ASTORB}^{3}$ database. Those values are in rough agreement with Waszczak et al. (2015) who reported $H_{B}$ and $G_{B}$ from over 54,000 asteroids observed in $g$ and $R$-band with the Palomar Transient Factory (PTF). They measured a mean value of $R_{P T F}=V_{M P C}+0.00$ which implies a systematic offset of $\sim 0.4 \mathrm{mag}$ in the MPC absolute magnitudes because the average $V-R$ for asteroids is $\sim 0.4$. Our values (fig. 8) are consistent with the MPC for $H_{B} \lesssim 11 \mathrm{mag}$ and $H_{B}>19 \mathrm{mag}$, i.e. within $\lesssim 0.1 \mathrm{mag}$ of the MPC absolute magnitudes (their reported precision), but are systematically higher than the MPC absolute magnitudes for $11 \mathrm{mag} \lesssim H_{B} \lesssim 19$ mag. i.e. our absolute magnitudes are systematically fainter than reported by the MPC and this would translate directly into predicting fainter apparent magnitudes than the MPC and, similarly, suggesting that the objects are smaller than predicted by the use of the MPC absolute magnitudes. The systematic difference reaches a maximum of $\sim 0.35 \mathrm{mag}$ at $H_{B} \sim 14$ in agreement with the earlier studies. This magnitude offset has implications for developing observing programs, selecting objects for followup, and for studies of the asteroids' size-frequency distribution.

\footnotetext{
${ }^{3}$ http://www.naic.edu/ nolan/astorb.html
} 


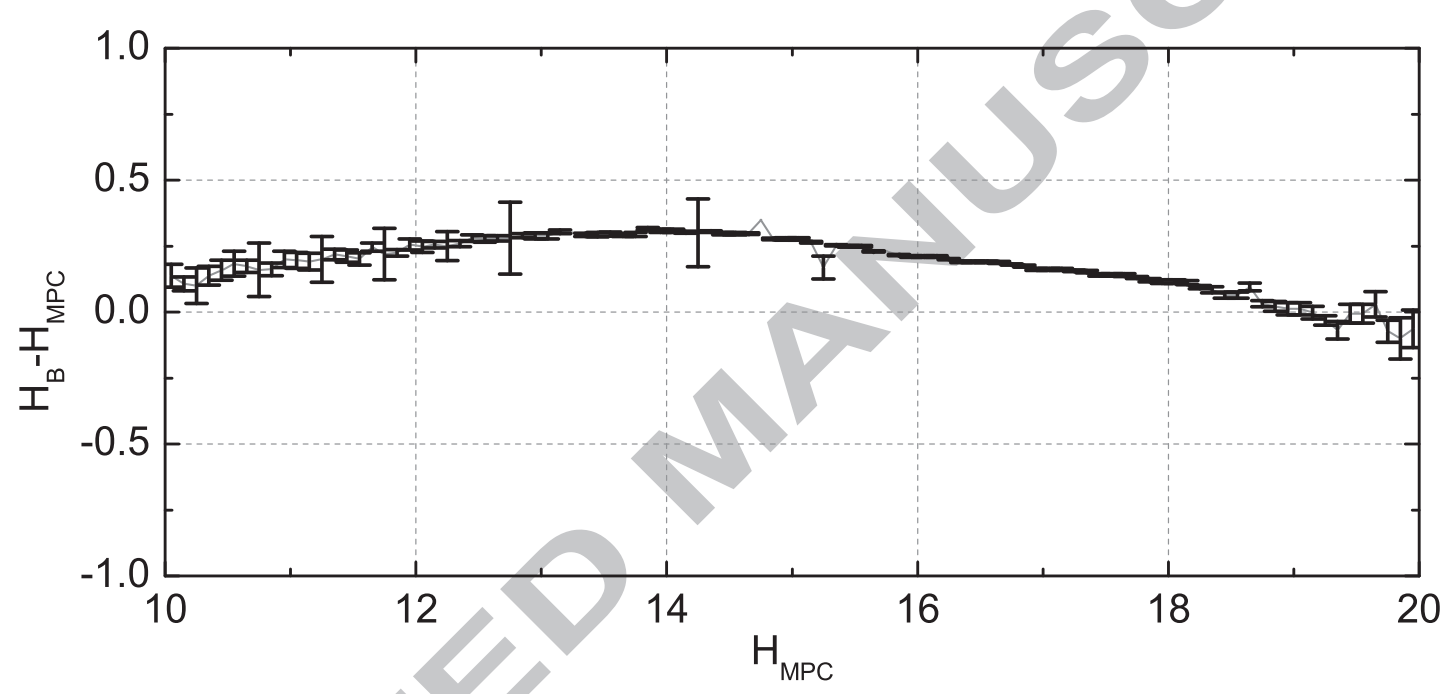

Fig. 8. - The difference between the absolute magnitude $H_{M P C}$ reported by the MPC using the B89 phase function and this work's $H_{B}$ value as a function of absolute magnitude. The thick solid black line represents the average in each 0.1 mag wide bin and the standard error on the mean is shown with error bars. The error bars are about the width of the line for $13 \lesssim H_{M P C} \lesssim 18$ 


\subsection{Slope Parameters}

The vast majority of Pan-STARRS1 asteroids offer only sparse phase angle coverage (Fig. 1) for the determination of the slope parameter but our MC technique should provide a realistic estimate of the statistical uncertainty and systematic error when the phase angle coverage is not too large and the detections are not in multiple apparitions.

The $G_{B}$ distribution (fig. 9 ) is very wide with a peak near 0.15 , the default slope parameter for objects of unknown spectral class (most of the asteroids in our sample). The distribution is artificially constrained between the lower and upper limits $\left(-0.25<G_{B}<0.8\right)$. On the other hand, the $G_{M}$ distribution has a broad peak centered on $G_{M} \sim 0.5$ superimposed on a roughly flat distribution of slope parameters between our artificial limits $\left(-0.5<G_{M}<1.5\right)$. The large peak near $G_{M}=0.2$ that contains $\sim 30 \%$ of all $G_{M}$ values is due to a discontinuity in the M10 phase function, it is not an error in our implementation. In comparison, $\sim 8 \%$ of the Oszkiewicz et al. (2012) $G_{M}$ values were also $\sim 0.2$. Our technique is particularly sensitive to the function discontinuity and has a propensity to drive the fitted $G_{M}$ value to 0.2 when the number of data points is small. We suggest that future attempts to use the M10 phase function flag and address this situation, perhaps by forcing $G_{M}=0.5$ in those cases.

The slope parameter uncertainty $(\delta G)$ distributions have peaks at zero corresponding to the $\sim 24 \%$ of cases in both methods where the MC technique did not converge and we fixed the slopes. Those $G_{B}$ that were actually fit have a normal-like distribution with mean $G_{B}=0.18 \pm 0.01$ and RMS of 0.05 (Fig. 9). Similarly, the $G_{M}$ uncertainty has a normal-like distribution with mean at $0.29 \pm 0.01$ and RMS of 0.17 . The $\delta G_{M}$ distribution is wider and shifted towards larger values than the $\delta G_{B}$ distribution because the $G_{M}$ values are fundamentally larger than the corresponding $G_{B}$ values. The percentage uncertainties $(\delta G /|G|$, fig. 9$)$ in both slope parameters are very similar, suggesting that the two phase 

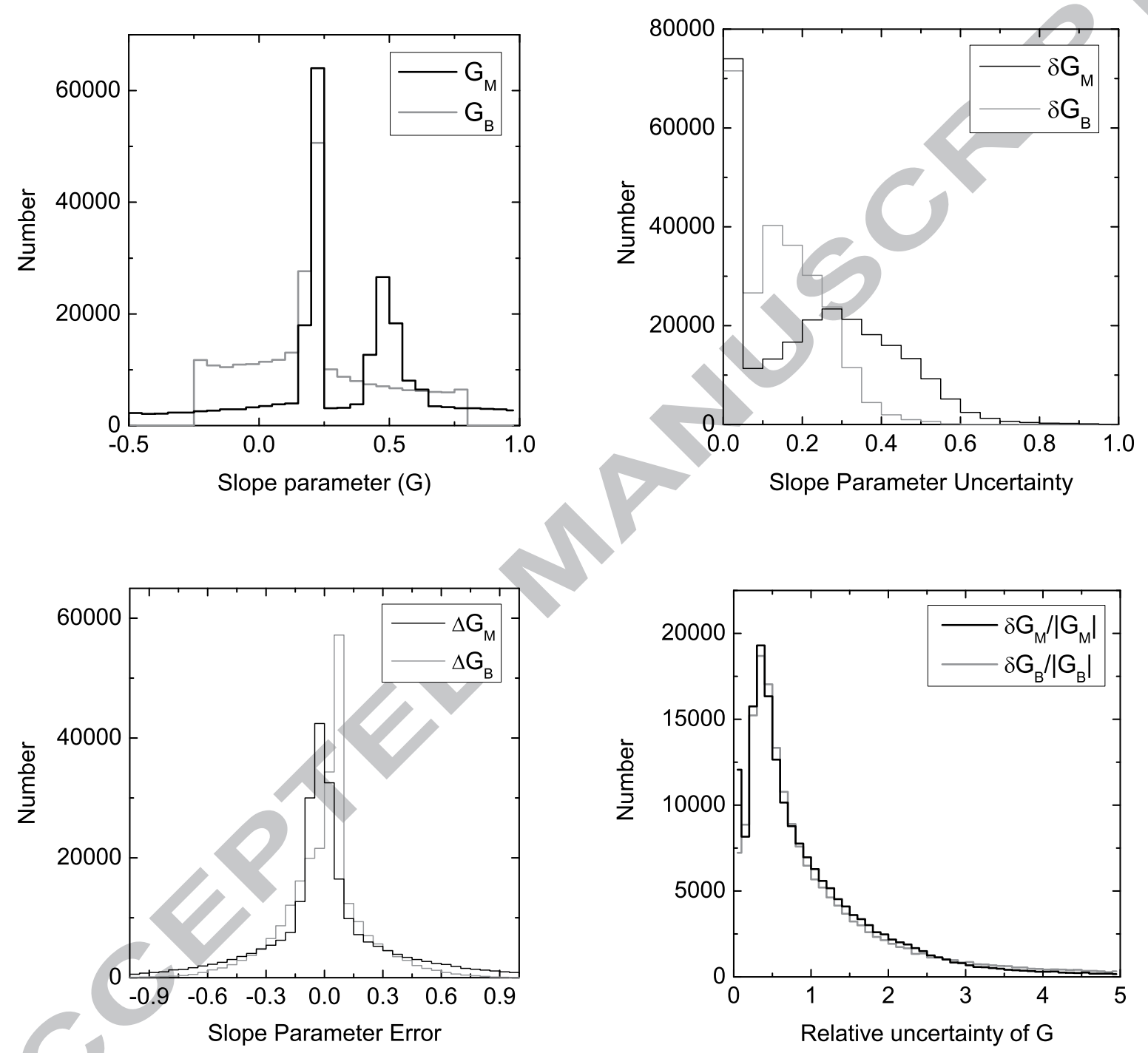

Fig. 9.- (top left) The slope parameters $G_{B}$ (B89) and $G_{M}$ (M10) and (top right) their uncertainties and (bottom left) errors. (bottom right) Percentage uncertainty in the slope parameter $(\delta G)$ with the (gray) B89 and (black) M10 photometric methods. 
functions are equally effective for calculating the slope parameters, at least in the regime applicable to this data sample. The mean relative slope parameter uncertainties are $\sim 34 \%$ and $\sim 0.36 \%$ for $G_{B}$ and $G_{M}$ respectively, the large values being due mostly to the limited phase curve coverage.
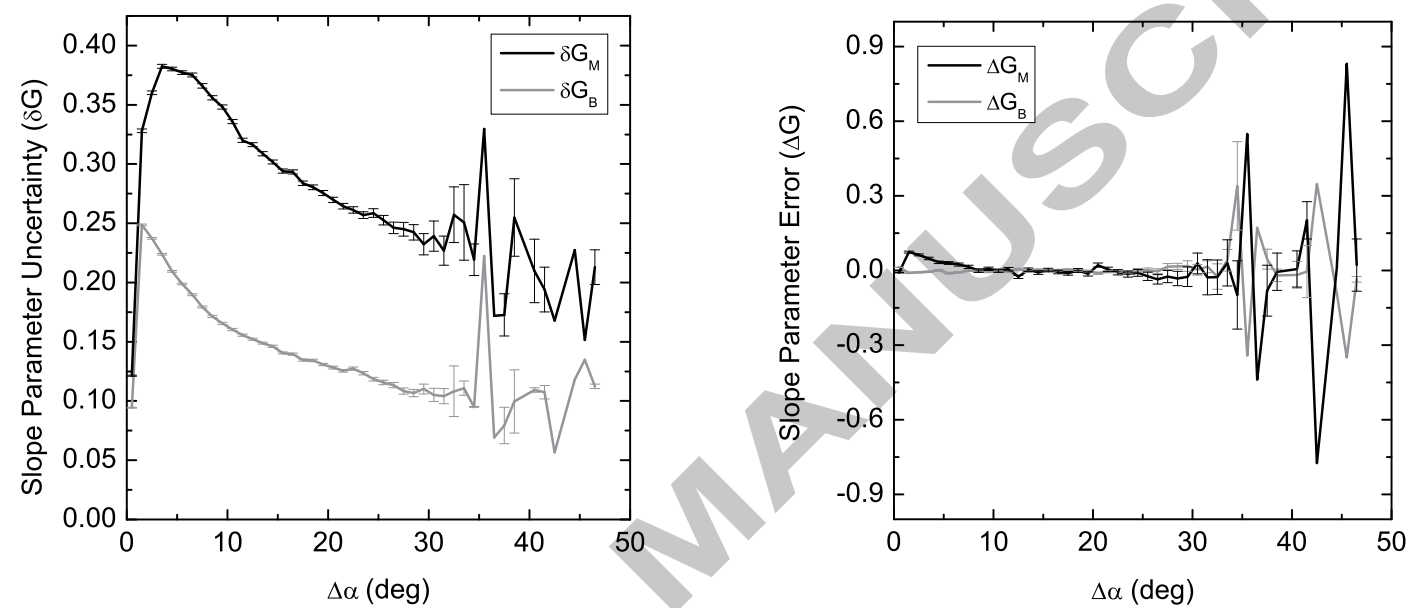

Fig. 10.- (left) Average MC slope parameter uncertainty and (right) error as a function of phase angle range using the (gray) B89 and (black) M10 phase functions.

As expected, the slope parameter uncertainty depends on the phase angle coverage $(\Delta \alpha$, fig. 10). The uncertainty is artificially small at small phase angle ranges near zero because in these cases the slope parameter was mostly fixed at a pre-specified value. The uncertainty is largest for $\Delta \alpha \sim 5^{\circ}$ because at this phase angle range the slope parameter begins to be calculable, and the uncertainty drops at larger phase-angle ranges because the data provides stronger constraints on the shape of the phase function. However, even in the best case scenario, for phase angle ranges of $\gtrsim 30 \mathrm{deg}$, the percentage uncertainty is still $\sim 50 \%$ for both phase functions. In any event, the number of objects in our data sample with large phase angle coverage is very small. Fig. 10 also illustrates that the systematic 
errors introduced by our MC technique are not dependent on phase angle coverage.

Pravec et al. (2012) provide acurate $G_{B}$ slope parameters with uncertainties for more than 500 asteroids with densely covered light curves in a single pass band over a wide range of phase angles. The mean difference between this work's $G_{B}$ and $G_{B, P R A}$ is $0.00 \pm 0.02$ with $\sigma \sim 0.28$ for the 196 asteroids in common between the two data sets with derived slope parameters (Fig. 11). The agreement between our MC solution and the accurate work of Pravec et al. (2012) using the B89 phase function suggests that our technique for calculating the slope parameter is viable for a large number of asteroids with sparsely sampled light curves. Furthermore, our technique allows us to estimate the mean error on the derived slope parameter, $\overline{\Delta G_{B}}=0.00 \pm 0.01$, so the MC technique does not introduce a systematic bias. The mean statistical uncertainty in the slope parameter for our data sample of $\overline{\delta G_{B}}=0.17 \pm 0.01$ is twice as large as the Pravec et al. (2012) data set of $\overline{\delta G_{B}}=0.09 \pm 0.01$ which could be interpreted as either surprisingly good, given the small number of observations and phase curve coverage of our data sample, or as an indication that measuring $G_{B}$ is difficult even with a very good data sample.

As described earlier, Oszkiewicz et al. (2012) derived asteroid slope parameters from photometry reported to the MPC from multiple observatories that used different filters and reference catalogs. They also had to deal with the fact that the MPC observation submission format did not allow reporting of photometric uncertainties. To reduce some of the associated problems they statistically calibrated the disparate datasets and used photometry only from major surveys. After excluding the artificial peak near $G_{M}=0.2$ (i.e., excluding the range $0.18<G_{M}<0.22$ ), and including only those objects for which $G$ was actually fit, there were 80,756 objects in common with our $G_{M}$ values and 133,884 objects for comparison with our $G_{B}$. The wide and oddly-shaped distribution of the difference in slope parameters between our MC technique and Oszkiewicz et al. (2012) 

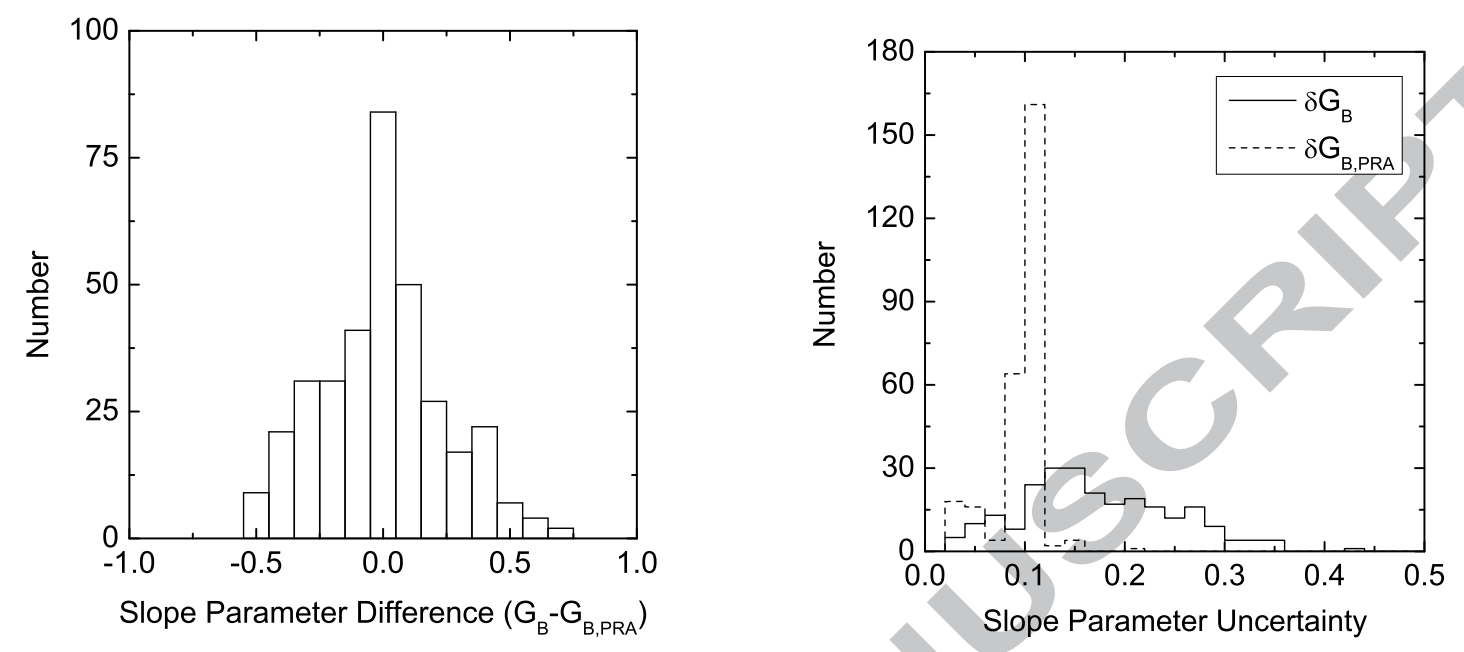

Fig. 11. - (left) The difference between our MC $G_{B}$ values (B89) and 196 objects in common with Pravec et al. (2012). (right) Slope parameter uncertainty distributions for the same 196 objects for (solid) our MC values and (dashed) Pravec et al. (2012).

(fig. 12) illustrates the difficulty and large uncertainty in measuring $G$. The distribution peaks at zero for the B89 phase function with $\left(\overline{G_{B}-G_{B, O S K}}=0.00 \pm 0.01\right)$ but there is a significant offset using the M10 phase function of $\overline{G_{M}-G_{M, O S K}}=-0.06 \pm 0.01$ (fig. 12). The RMS of the difference is larger using the M10 (0.58) than with the B89 phase function (0.35) but this is expected due to the numerically larger expected values of $G_{M} \sim 0.5$.

Fig. 12 also illustrates that our MC technique yields slope parameters that are comparable or marginally better than the work of Oszkiewicz et al. (2012), even though our data sample includes much less photometric data per object over a narrower phase angle range, presumably because of the Pan-STARRS1 system's superior photometry and the use of measured photometric uncertainties. The mean uncertainty for 80,756 objects in common with Oszkiewicz et al. (2012) is $0.33 \pm 0.01(\mathrm{RMS}=0.14)$ with our MC technique and is $0.39 \pm 0.01(\mathrm{RMS}=0.18)$ for the values reported by Oszkiewicz et al. (2012). 

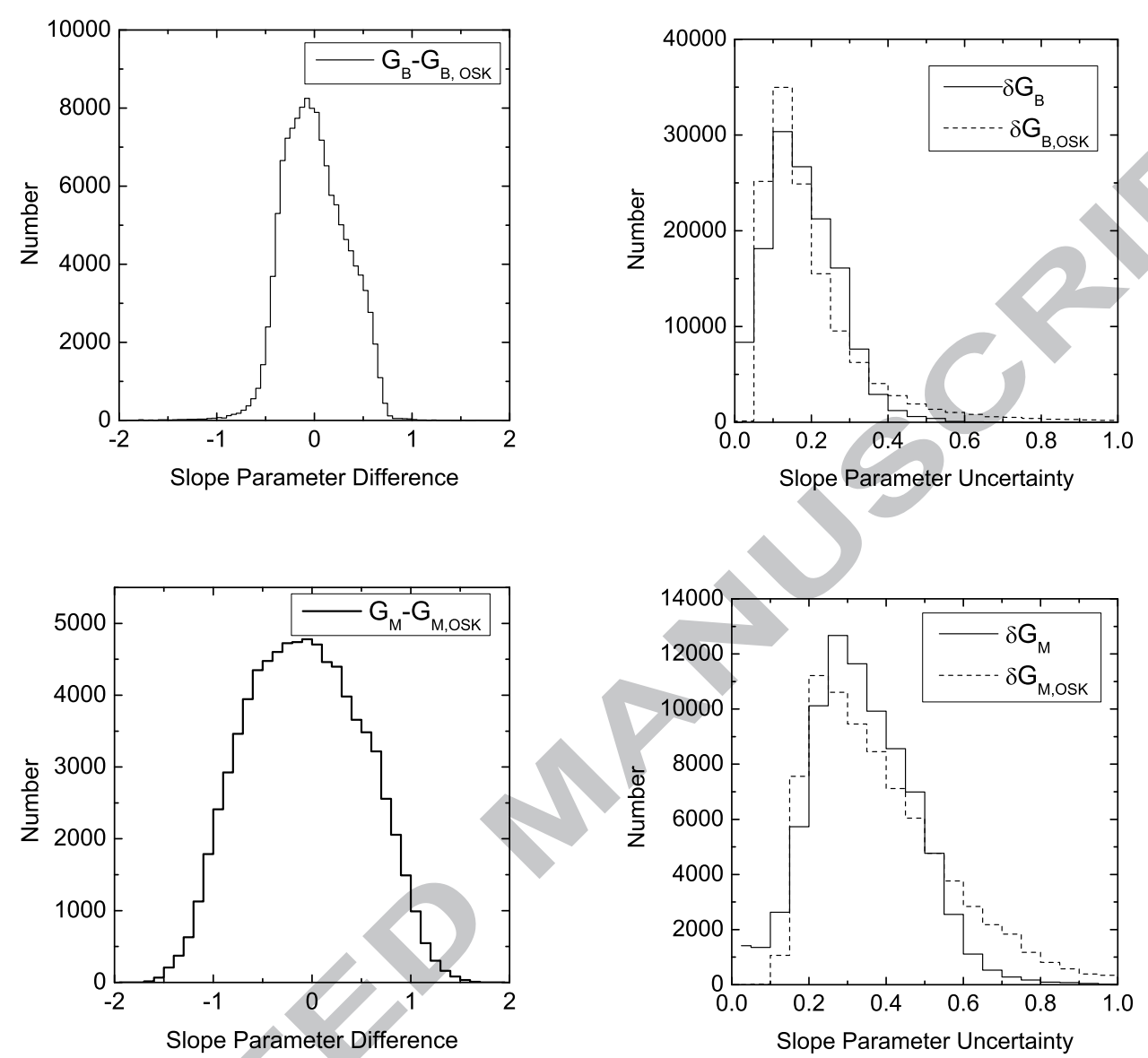

Fig. 12.- (top left) Difference between our MC $G_{B}$ values (M10) and 133,885 objects in common with Oszkiewicz et al. (2012). (top right) Slope parameter uncertainties for the same objects as determined in this work and by Oszkiewicz et al. (2012). (bottom left) Difference between our MC $G_{M}$ values (M10) and 80,756 objects in common with Oszkiewicz et al. (2012). (bottom right) Slope parameter uncertainties for the same objects as determined in this work and by Oszkiewicz et al. (2012).

Slope parameters are taxonomy-dependent (Harris 1989; Lagerkvist and Magnusson 1990; Oszkiewicz et al. 2012; Pravec et al. 2012) but most of the objects in our Pan-STARRS1 data sample are fainter than known asteroids with well established taxonomies, so we relied 
Table 5: Mean slope parameters \pm standard deviation $\left(G_{B}\right.$, B89) derived in this work (PS1, second column) and by Pravec et al. (2012) (PRA12, third column) for the same objects in 4 major taxonomic classes. The last column is the number of common objects that have a Hasselmann et al. (2012) spectral classification (no D type asteroids satisfied our requirements on taxonomic identification and slope parameter determination).

\begin{tabular}{c|cc|c}
\hline \hline $\begin{array}{c}\text { Taxonomic } \\
\text { Class }\end{array}$ & $\begin{array}{c}G_{B} \\
\text { PS1 }\end{array}$ & $\begin{array}{c}G_{B} \\
\text { PRA12 }\end{array}$ & N \\
\hline $\mathrm{Q}$ & $\mathbf{0 . 1 1} \pm \mathbf{0 . 1 6}$ & $0.19 \pm 0.10$ & 3 \\
$\mathrm{~S}$ & $\mathbf{0 . 1 6} \pm \mathbf{0 . 2 6}$ & $0.23 \pm 0.05$ & 32 \\
$\mathrm{C}$ & $\mathbf{0 . 0 3} \pm \mathbf{0 . 1 0}$ & $0.13 \pm 0.01$ & 4 \\
$\mathrm{D}$ & $\mathbf{n} / \mathbf{a}$ & $\mathrm{n} / \mathrm{a}$ & 0 \\
$\mathrm{X}$ & $\mathbf{0 . 2 1} \pm \mathbf{0 . 3 0}$ & $0.20 \pm 0.10$ & 9 \\
\hline \hline
\end{tabular}

on the SDSS spectral classification (Hasselmann et al. 2012) to assess our method's ability to detect the taxonomic-dependence. We found 48 asteroids in common with Pravec et al. (2012) and 18,541 with Oszkiewicz et al. (2012) (excluding values around $G_{M} \sim 0.20$ ) for which we could compare our calculated slope parameters. Our mean \pm RMS $G_{B}$ values are consistent with Pravec et al. (2012) (Table 4.3) but our RMS distribution is much larger and the common number of asteroids is very low. Similarly, our $G_{B}$ and $G_{M}$ values (Table 6) are consistent Oszkiewicz et al. (2012) but the RMS is distributions are large in both cases. 
Table 6: Slope parameters derived in this work (PS1: $G_{B}$, second column; $G_{M}$ fifth column) and by Oszkiewicz et al. (2012) (OSK12: $G_{B}$, third column; $G_{M}$, sixth column) for the same objects in five different spectral classes. The forth and last columns are the number of objects in common between the two data sets with SDSS spectral classification (Carvano et al. 2010).

\begin{tabular}{|c|c|c|c|c|c|c|}
\hline Taxonomic & $G_{B}$ & $G_{B}$ & $\mathrm{~N}$ & $G_{M}$ & $G_{M}$ & $\mathrm{~N}$ \\
\hline Class & PS1 & OSK12 & & PS1 & OSK12 & \\
\hline $\mathrm{Q}$ & $0.21 \pm 0.28$ & $0.20 \pm 0.10$ & 1324 & $0.46 \pm 0.53$ & $0.54 \pm 0.22$ & 886 \\
\hline S & $0.22 \pm 0.28$ & $0.19 \pm 0.22$ & 14686 & $0.47 \pm 0.53$ & $0.55 \pm 0.20$ & 10231 \\
\hline $\mathrm{C}$ & $0.18 \pm 0.28$ & $0.16 \pm 0.10$ & 7892 & $0.58 \pm 0.55$ & $0.66 \pm 0.23$ & 5150 \\
\hline $\mathrm{D}$ & $0.23 \pm 0.29$ & $0.19 \pm 0.12$ & 1321 & $0.42 \pm 0.52$ & $0.61 \pm 0.25$ & 852 \\
\hline $\mathrm{X}$ & $0.19 \pm 0.28$ & $0.18 \pm 0.11$ & 2073 & $0.53 \pm 0.54$ & $0.59 \pm 0.24$ & 1428 \\
\hline
\end{tabular}

There is a formal difference between the means of some of the taxonomic classes but we do not consider them further because of the large uncertainties on each value and the large RMS of each taxonomic class' $G$ distribution.

As discussed above in section 3.1, the phase curve coefficients $G_{B}$ and $G_{M}$ are functions of asteroid composition. Given the compositional trends of the inner belt being dominated by silicate S-type asteroids and carbon/volatile-rich asteroids in the outer belt, we should expect to see these trends reflected in our derived phase functions. A similar study was performed by Oszkiewicz et al. (2012) in their analysis of the MPC database. They found correlations between their measured G12 and orbital elements throughout the main belt, reflecting the general compositional gradient and family structure. To study this in our database we selected the 51,864 asteroids with orbital semi-major axes $2.1 \leq a \leq 3.3 \mathrm{AU}$ 
where the range of phase angles observed was $\Delta \alpha \geq 5^{\circ}$ and there were $N \geq 6$ observations. We then calculated the running median values $\overline{G_{B}}$ and $\overline{G_{M}}$ as a function of orbital $a$ over a range $\Delta a=0.05 \mathrm{AU}$.

Figure 13 clearly shows clear a negative trend in $\overline{G_{B}}$ and a positive trend in $\overline{G_{M}}$ with orbital $a$. As $G_{B}$ is larger for S-type than C-type asteroids, while $G_{M}$ becomes smaller, this agrees with the established compositional gradient in the main-belt. For modelling purposes, these trends may be approximated by the relationships $\bar{G}_{B}=-0.103 a+0.446$ and $\overline{G_{M}}=0.237 a-0.175$ within the main belt. The largest deviations from these relationships occur at the 3:1 Kirkwood gap at 2.50 AU, and at the 7:3 gap at 2.95 AU. This latter position marks where the S-type asteroids of the dominant Koronis family of gives way to the T/X/K/D-type asteroids of the Eos family (Mothé-Diniz et al. 2005). We note that the overall observed scatter in individual values is dominated by $\Delta G$, although it will also be partly due to the large amount of compositional mixing present in the main belt (DeMeo and Carry 2013). 

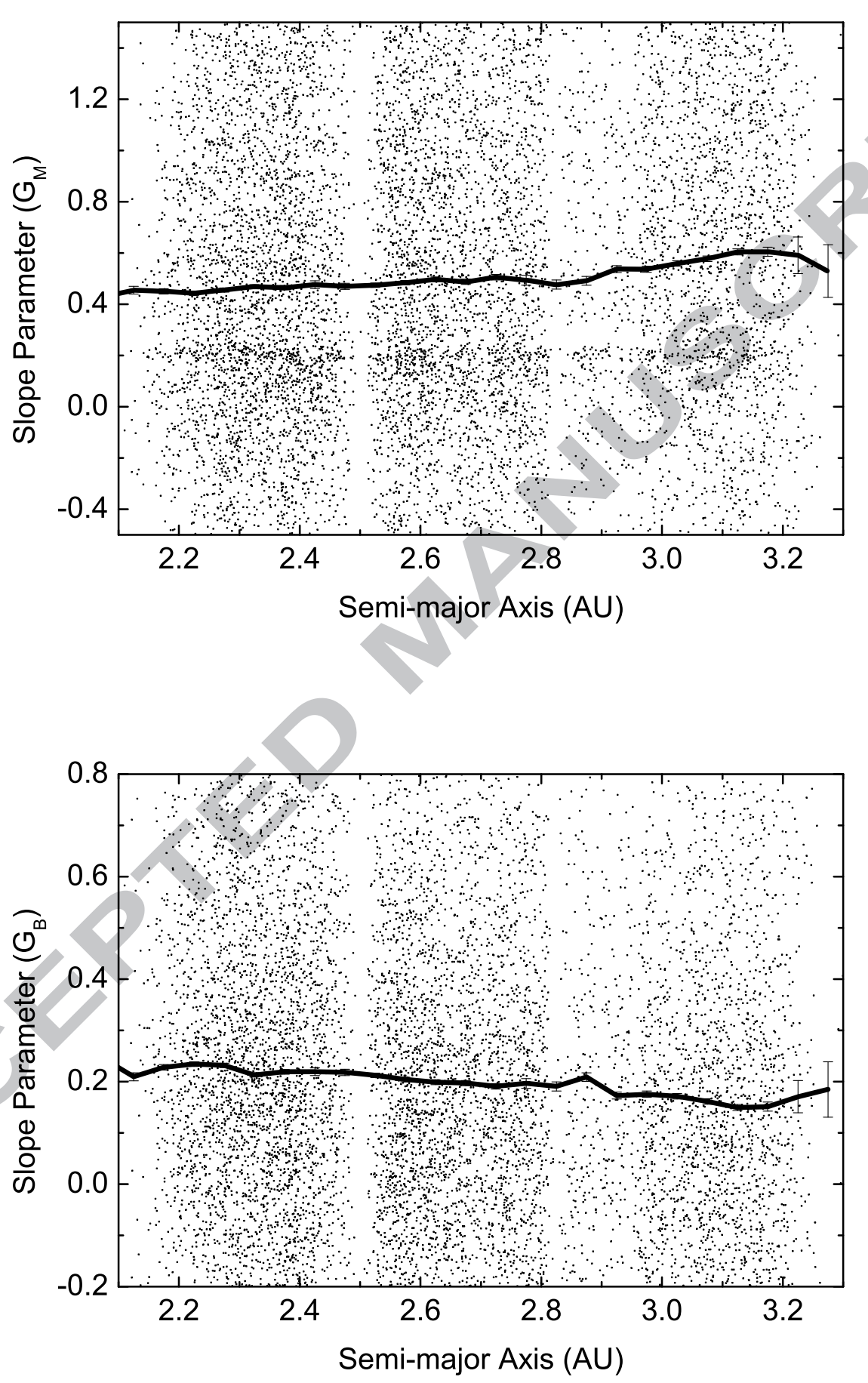

Fig. 13.- Moving average of $G_{M}$ (top) and $G_{B}$ (bottom) as a function semi-major axis. 


\section{Availability}

The Pan-STARRS1 absolute magnitudes and slope parameters with associated uncertainties as described herein are available on-line (Appendix A). The eventual goal is that the catalog will be updated with all the data from the entire 3 year Pan-STARRS1 mission and then updated regularly with new data from the ongoing extended mission that is purely focused on the solar system. This effort will provide almost complete coverage of all known asteroids with extensive phase angle coverage and good number of detections per object.

\section{Conclusions}

Our work introduces a Monte Carlo method for calculating absolute magnitudes $(H)$ and slope parameters $(G)$ and their statistical uncertainties and systematic errors that is applicable to single apparition asteroid observations and designed to handle limited photometric data over a restricted phase angle range. The technique's utility was confirmed by comparing our $H$ and $G$ values to the well-established results of Pravec et al. (2012) for a limited number of objects. We then applied it to derive $H$ and $G$ with statistical uncertainties and systematic errors for $\sim 240,000$ numbered asteroids observed in the first 15 months of Pan-STARRS1's 3-year nominal mission. The single-survey data, consistent image processing, and well-defined photometric calibration, eliminates many of the problems encountered in past attempts to measure absolute magnitudes and slope parameters from a combination of different surveys.

We find that the Muinonen et al. (2010) phase function provides better results than the Bowell et al. (1989) phase function in terms of reducing the statistical uncertainty and systematic error on the absolute magnitude — both crucial to accurately predicting 
ephemeris apparent magnitudes and calculating asteroid albedos from $H$ and measured asteroid diameters. There is a systematic $H$-dependent offset between the Minor Planet Center's reported absolute magnitude and $H$ derived in this work with a maximum offset of about $0.25 \mathrm{mag}$ at $H \sim 14$.

The measured slope parameters are generally in agreement with the results of Pravec et al. (2012) and Oszkiewicz et al. (2012) but the statistical uncertainty and systematic error on any individual asteroid's $G$ is large due to poor temporal and phase-space coverage.

\section{Acknowledgements}

The Pan-STARRS1 Surveys have been made possible through contributions of the Institute for Astronomy, the University of Hawaii, the Pan-STARRSProject Office, the Max-Planck Society and its participating institutes, the Max Planck Institute for Astronomy, Heidelberg and the Max Planck Institute for Extraterrestrial Physics, Garching, The Johns Hopkins University, Durham University, the University of Edinburgh, Queen's University Belfast, the Harvard-Smithsonian Center for Astrophysics, and the Las Cumbres Observatory Global Telescope Network, Incorporated, the National Central University of Taiwan, and the National Aeronautics and Space Administration under Grant No. NNX08AR22G and No. NNX12AR65G issued through the Planetary Science Division of the NASA Science Mission Directorate. We would like to thank two anonymous reviewers for their constructive feedback. 


\section{A. Pan-STARRS1 asteroid database}

Version 1.0 of the Pan-STARRS1 asteroid database is available at http://www.ifa.hawaii.edu/NEO/ It provides derived $H$ and $G$ values for 248,457 asteroids with a total of 1,242,282 detections spanning the time interval from February 2011 to May 2012 as described in this work. The 18 column data file is comma-delimited and each line represents a single asteroid. The columns are described in table 7. 


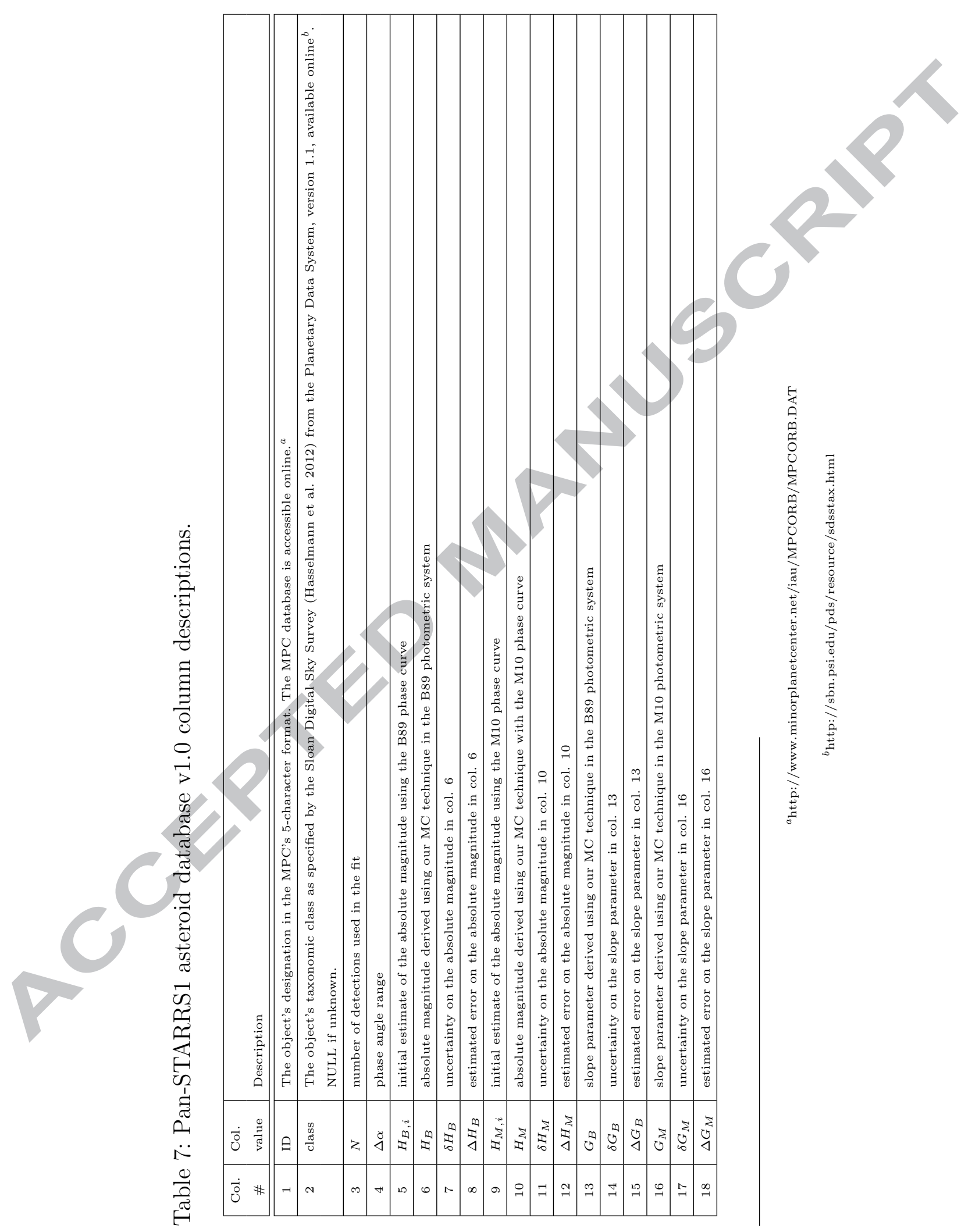




\section{REFERENCES}

Bowell, E., Happke, B., Domingue, D., Lumme, K., Peltoniemi, J., Harris, A., 1989. In: Gehrels, T., Matthews, M. T., Binzel, R. P. (Eds.), Asteroids III. University of Arizona Press, Chapter Application of photometric models to asteroids, pp. 524-555.

Carvano, J. M., Hasselmann, P. H., Lazzaro, D., Mothé-Diniz, T., 2010. SDSS-based taxonomic classification and orbital distribution of main belt asteroids. A\&A 510, A43.

de la Fuente Marcos, C., de la Fuente Marcos, R., 2013. A resonant family of dynamically cold small bodies in the near-Earth asteroid belt. MNRAS 434, L1-L5.

DeMeo, F. E., Carry, B., 2013. The taxonomic distribution of asteroids from multi-filter all-sky photometric surveys. 226 (1), 723-741.

Denneau, L., Jedicke, R., Grav, T., Granvik, M., Kubica, J., Milani, A., Vereš, P., Wainscoat, R., Chang, D., Pierfederici, F., Kaiser, N., Chambers, K. C., Heasley, J. N., Magnier, E. A., Price, P. A., Myers, J., Kleyna, J., Hsieh, H., Farnocchia, D., Waters, C., Sweeney, W. H., Green, D., Bolin, B., Burgett, W. S., Morgan, J. S., Tonry, J. L., Hodapp, K. W., Chastel, S., Chesley, S., Fitzsimmons, A., Holman, M., Spahr, T., Tholen, D., Williams, G. V., Abe, S., Armstrong, J. D., Bressi, T. H., Holmes, R., Lister, T., McMillan, R. S., Micheli, M., Ryan, E. V., Ryan, W. H., Scotti, J. V., 2013. The Pan-STARRS Moving Object Processing System. PASP 125, $357-395$.

Fukugita, M., Ichikawa, T., Gunn, J. E., Doi, M., Shimasaku, K., Schneider, D. P., 1996. The Sloan Digital Sky Survey Photometric System. AJ 111, 1748.

Grav, T., Mainzer, A. K., Bauer, J., Masiero, J., Spahr, T., McMillan, R. S., Walker, R., Cutri, R., Wright, E., Eisenhardt, P. R., Blauvelt, E., DeBaun, E., Elsbury, 
D., Gautier, T., Gomillion, S., Hand, E., Wilkins, A., 2012. WISE/NEOWISE Observations of the Hilda Population: Preliminary Results. ApJ 744, 197.

Grav, T., Mainzer, A. K., Bauer, J. M., Masiero, J. R., Nugent, C. R., 2012. WISE/NEOWISE Observations of the Jovian Trojan Population: Taxonomy. ApJ 759, 49.

Harris, A. W., 1989. The H-G Asteroid Magnitude System: Mean Slope Parameters. In: Lunar and Planetary Institute Science Conference Abstracts, Volume 20 of Lunar and Planetary Inst. Technical Report, pp. 375.

Hasselmann, P., Carvano, J. M., Lazzaro, D., 2012. Sdss-based asteroid taxonomy v1.1. ear-a-i0035-5-sdsstax-v1.1.

Holsapple, K. A., 2007. Spin limits of Solar System bodies: From the small fast-rotators to 2003 EL61. Icarus 187, 500-509.

Jedicke, R., Larsen, J., Spahr, T., 2002. Observational Selection Effects in Asteroid Surveys. Asteroids III, $71-87$.

Jurić, M., Ivezić, Ž., Lupton, R. H., Quinn, T., Tabachnik, S., Fan, X., Gunn, J. E., Hennessy, G. S., Knapp, G. R., Munn, J. A., Pier, J. R., Rockosi, C. M., Schneider, D. P., Brinkmann, J., Csabai, I., Fukugita, M., 2002. Comparison of Positions and Magnitudes of Asteroids Observed in the Sloan Digital Sky Survey with Those Predicted for Known Asteroids. AJ 124, 1776-1787.

Kaiser, N., Burgett, W., Chambers, K., Denneau, L., Heasley, J., Jedicke, R., Magnier, E., Morgan, J., Onaka, P., Tonry, J., 2010. The pan-starrs wide-field optical/nir imaging survey. In: Stepp L.M., G. R., H.J., H. (Eds.), Ground-based and Airborne Telescopes III, Volume 7732 of Proceedings of the SPIE, pp. 77330E-77330E-14. 
Lagerkvist, C.-I., Magnusson, P., 1990. Analysis of asteroid lightcurves. II - Phase curves in a generalized HG-system. A\&AS 86, 119-165.

Law, N. M., Kulkarni, S. R., Dekany, R. G., Ofek, E. O., Quimby, R. M., Nugent, P. E., Surace, J., Grillmair, C. C., Bloom, J. S., Kasliwal, M. M., Bildsten, L., Brown, T., Cenko, S. B., Ciardi, D., Croner, E., Djorgovski, S. G., van Eyken, J., Filippenko, A. V., Fox, D. B., Gal-Yam, A., Hale, D., Hamam, N., Helou, G., Henning, J., Howell, D. A., Jacobsen, J., Laher, R., Mattingly, S., McKenna, D., Pickles, A., Poznanski, D., Rahmer, G., Rau, A., Rosing, W., Shara, M., Smith, R., Starr, D., Sullivan, M., Velur, V., Walters, R., Zolkower, J., 2009. The Palomar Transient Factory: System Overview, Performance, and First Results. PASP 121, 1395-1408.

Levenberg, K., 1944. A method for the solution of certain problems in least squares. Quarterly of Applied Mathematics 2, 164-168.

Lupton, R., 2007. The characterization, subtraction, and addition of astronomical images. In: G.J., B., E.D., F. (Eds.), Statistical Challenges in Modern Astronomy IV, Volume 371 of ASP Conference Series, pp. 160-172.

Magnier, E., 2006. The Pan-STARRS PS1 Image Processing Pipeline. In: The Advanced Maui Optical and Space Surveillance Technologies Conference.

Magnier, E. A., Schlafly, E., Finkbeiner, D., Juric, M., Tonry, J. L., Burgett, W. S., Chambers, K. C., Flewelling, H. A., Kaiser, N., Kudritzki, R.-P., Morgan, J. S., Price, P. A., Sweeney, W. E., Stubbs, C. W., 2013. The Pan-STARRS 1 Photometric Reference Ladder, Release 12.01. ApJS 205, 20.

Marquardt, D., 1963. An algorithm for least-squares estimation of nonlinear parameters. SIAM Journal on Applied Mathematics 11, 431-441. 
Masiero, J., Jedicke, R., Ďurech, J., Gwyn, S., Denneau, L., Larsen, J., 2009. The Thousand Asteroid Light Curve Survey. Icarus 204, 145-171.

Milani, A., Gronchi, G. F., Farnocchia, D., Knežević, Z., Jedicke, R., Denneau, L., Pierfederici, F., 2008. Topocentric orbit determination: Algorithms for the next generation surveys. Icarus 195, 474-492.

Mothé-Diniz, T., Carvano, J. M. Á., Lazzaro, D., 2003. Distribution of taxonomic classes in the main belt of asteroids. Icarus 162, 10-21.

Mothé-Diniz, T., Roig, F., Carvano, J. M., 2005. Reanalysis of asteroid families structure through visible spectroscopy. Icarus $174(1), 54-80$.

Muinonen, K., Belskaya, I., Cellino, A., Delbo, M., Levasseur-Regourd, A.-C., Penttilä, A., Tedesco, E., 2010. A three-parameter magnitude phase function for asteroids. Icarus 209, 542-555.

Oszkiewicz, D. A., Bowell, E., Wasserman, L. H., Muinonen, K., Penttilä, A., Pieniluoma, T., Trilling, D. E., Thomas, C. A., 2012. Asteroid taxonomic signatures from photometric phase curves. Icarus 219, 283-296.

Parker, A., Ivezić, Z̆., Jurić, M., Lupton, R., Sekora, M. D., Kowalski, A., 2008. The size distributions of asteroid families in the SDSS Moving Object Catalog 4. Icarus 198, $138-155$.

Pravec, P., Harris, A. W., Kušnirák, P., Galád, A., Hornoch, K., 2012. Absolute magnitudes of asteroids and a revision of asteroid albedo estimates from WISE thermal observations. Icarus 221, 365-387.

Schlafly, E. F., Finkbeiner, D. P., Jurić, M., Magnier, E. A., Burgett, W. S., Chambers, K. C., Grav, T., Hodapp, K. W., Kaiser, N., Kudritzki, R.-P., Martin, N. F., 
Morgan, J. S., Price, P. A., Rix, H.-W., Stubbs, C. W., Tonry, J. L., Wainscoat, R. J., 2012. Photometric Calibration of the First 1.5 Years of the Pan-STARRS1 Survey. ApJ 756, 158.

Stuart, J. S., Binzel, R. P., 2004. Bias-corrected population, size distribution, and impact hazard for the near-Earth objects. Icarus 170, 295-311.

Tonry, J., Onaka, P., 2009. The Pan-STARRS Gigapixel Camera. In: Advanced Maui Optical and Space Surveillance Technologies Conference.

Tonry, J. L., Stubbs, C. W., Lykke, K. R., Doherty, P., Shivvers, I. S., Burgett, W. S., Chambers, K. C., Hodapp, K. W., Kaiser, N., Kudritzki, R.-P., Magnier, E. A., Morgan, J. S., Price, P. A., Wainscoat, R. J., 2012. The Pan-STARRS1 Photometric System. ApJ 750, 99.

Warner, B. D., Harris, A. W., Pravec, P., 2009. The asteroid lightcurve database. Icarus 202, $134-146$.

Waszczak, A., Chang, C.-K., Ofek, E. O., Laher, R., Masci, F., Levitan, D., Surace, J., Cheng, Y.-C., Ip, W.-H., Kinoshita, D., Helou, G., Prince, T. A., Kulkarni, S., 2015. Asteroid lightcurves from the Palomar Transient Factory survey: Rotation periods and phase functions from sparse photometry. ArXiv e-prints.

Wiegert, P. A., Innanen, K. A., Mikkola, S., 1997. An asteroidal companion to the Earth. Nature $387,685-686$.

Zavodny, M., Jedicke, R., Beshore, E. C., Bernardi, F., Larson, S., 2008. The orbit and size distribution of small Solar System objects orbiting the Sun interior to the Earth's orbit. Icarus 198, 284-293.

This manuscript was prepared with the AAS LATEX macros v5.2. 


\section{Highlights}

We calculated absolute magnitudes and slope parameters of 240,000 asteroids

We provided uncertainties and errors on derived values

Photometry from the Pan-STARRS1 survey was used in our work

We employed both Bowell's (1989) and Muinonen's (2010) phase functions

We found discrepancy between our absolute magnitudes and those derived by MPC 